\title{
$H$ (div) PRECONDITIONING FOR A MIXED FINITE ELEMENT FORMULATION OF THE DIFFUSION PROBLEM WITH RANDOM DATA
}

\author{
HOWARD C. ELMAN, DARRAN G. FURNIVAL, AND CATHERINE E. POWELL
}

\begin{abstract}
We study $H($ div) preconditioning for the saddle-point systems that arise in a stochastic Galerkin mixed formulation of the steady-state diffusion problem with random data. The key ingredient is a multigrid V-cycle for an $H$ (div) operator with random weight function acting on a certain tensor product space of random fields with finite variance. We build on the ArnoldFalk-Winther multigrid algorithm presented in 1997 by varying the spatial discretization from grid to grid whilst keeping the stochastic discretization fixed. We extend the deterministic analysis to accommodate the modified $H$ (div) operator and establish spectral equivalence bounds with a new multigrid V-cycle operator that are independent of the spatial and stochastic discretization parameters. We implement multigrid within a block-diagonal preconditioner for the full saddle-point problem, derive eigenvalue bounds for the preconditioned system matrices and investigate the impact of all the discretization parameters on the convergence rate of preconditioned MINRES.
\end{abstract}

\section{INTRODUCTION}

In deterministic modeling of physical processes, input variables that represent material properties, boundary conditions, or source terms are assumed to be known explicitly. This results in deterministic partial differential equations (PDEs) whose numerical solution can be effected via traditional discretization methods. In situations where complete knowledge of an input variable is lacking, it is more fitting to pose the problem in a probabilistic setting and solve the resulting stochastic PDEs. Note that by stochastic PDEs, we mean PDEs with random data as opposed to the stochastic PDEs encountered in stochastic analysis that are PDEs perturbed by white noise. The present work is concerned with the numerical solution of the steady-state diffusion equation with random data, written in mixed form. Here, the source of uncertainty is the diffusion coefficient which is modeled as a random field with a prescribed mean and correlation function.

In the last few years there has been a great deal of interest in so-called stochastic Galerkin methods for solving elliptic PDEs with random field coefficients (e.g., see [7, 2], 23]). This body of work has developed rigorous analysis for the pioneering work 12 which advocates coupling a polynomial chaos basis for a subspace of random functions with finite variance, with a traditional finite element spatial

Received by the editor June 26, 2008 and, in revised form, February 27, 2009.

2010 Mathematics Subject Classification. Primary 65F08; Secondary 35R60.

This work was supported by the U. S. Department of Energy under grant DEFG0204ER25619, and by the U. S. National Science Foundation undergrant CCF0726017.

(C)2009 American Mathematical Society Reverts to public domain 28 years from publication 
discretization. The advantage of this so-called stochastic finite element methodology is that a single linear system needs to be solved, the solution to which can be post-processed to obtain probabilistic information of interest. However, this system is typically orders of magnitude larger than those obtained from corresponding deterministic models, and specialized solvers are essential.

If the Galerkin discretization of the stochastic PDE in question is based on a primal weak formulation, the resulting linear system is symmetric and positive definite. An efficient multigrid solver for those systems was proposed in [14 and 8. If the discretization is based on a mixed formulation, then we have to solve a large-scale symmetric, indefinite system of equations, which is more challenging. A mean-based preconditioning scheme was suggested in [9. However, it loses efficiency if the variance of the random field coefficients is large relative to the mean field.

In [1], Arnold et al. pointed out that the saddle-point matrices that arise from mixed finite element discretizations of the deterministic steady-state diffusion equation (with unit coefficients) have the same mapping properties as a block-diagonal matrix whose blocks are discrete representations of the deterministic $H$ (div) and $L^{2}$ operators. If exact solves are assumed, this matrix is known to provide a preconditioner for MINRES that yields convergence independent of the spatial discretisation parameter. Computing the action of the inverse of the leading block is not a trivial task, however, and so a multigrid algorithm for $H(\operatorname{div})$ operators was suggested, leading to a practical preconditioner. Other authors have also suggested deterministic $H$ (div) multigrid algorithms (e.g., see [15, 25, 13]). The work of Arnold et al. [1] is attractive, however, since it takes the form of a standard multigrid V-cycle method with a specialized smoother.

In this work, we propose and analyze a new $H$ (div) multigrid method. We extend the deterministic analysis from [1] to accommodate an $H$ (div) operator which is weighted by random diffusion coefficients and we establish spectral equivalence bounds with the proposed multigrid V-cycle operator that are totally independent of the spatial and stochastic discretization parameters. We implement multigrid within a block-diagonal preconditioner for the full saddle-point system and investigate the impact of all the discretization parameters on the convergence rate of preconditioned MINRES. Numerical results are presented in 88 and we include experiments with a cheaper variant of the analyzed method.

\section{Steady-State Diffusion PRoblem With RANDom Data}

Here we introduce the stochastic PDE to be solved, derive a weak mixed formulation and an inf-sup stable finite-dimensional problem. As noted in $\$ 1$, the finite-dimensional problem is obtained by application of a Galerkin finite element method with the solution variables being expressed using curtailed polynomial chaos expansions. A semi-discrete formulation of the problem, which will be used in the subsequent analysis, is also introduced.

2.1. Boundary value problem. The stochastic steady-state diffusion equation with homogeneous Dirichlet boundary value conditions is given by

$$
\begin{cases}-\nabla \cdot(c \nabla p)=f & \text { in } D \times \Omega, \\ p=0 & \text { on } \partial D \times \Omega,\end{cases}
$$

where $D$ is the spatial domain, $\Omega$ is a sample space, $c: D \times \Omega \rightarrow \mathbb{R}$ is the diffusion coefficient, and $f: D \times \Omega \rightarrow \mathbb{R}$ is the source function. The sample space in turn 
belongs to a probability space $(\Omega, \mathcal{F}, P)$, where $\mathcal{F}$ is a $\sigma$-algebra and $P$ is a probability measure. Note that the divergence and gradient operators are considered to act on spatial components only. We will consider the spatial domain, $D$, to be a convex two-dimensional bounded open set with piecewise smooth boundary. In particular, we take $D$ to be the interior of a polygon.

Often in solving the diffusion problem the quantity $c \nabla p$ is of more interest than the quantity $p$. Instead of seeking an approximation to $p$ in (1) and then postprocessing this to obtain an approximation to $c \nabla p$, a preferred approach is to obtain an approximation for $c \nabla p$ directly by converting the second-order system to a first-order system. This is achieved by making the substitution $\vec{u}=c \nabla p$ which gives

$$
\begin{cases}c^{-1} \vec{u}-\nabla p=0 & \text { in } D \times \Omega, \\ -\nabla \cdot \vec{u}=f & \text { in } D \times \Omega, \\ p=0 & \text { on } \partial D \times \Omega .\end{cases}
$$

We will refer to $p$ as the displacement and $\vec{u}$ as the flux.

In order to characterize the random aspect of the problem, we start by assuming that $c^{-1}$ is expressed as a curtailed Karhunen-Loève expansion, i.e.

$$
c(\vec{x}, \vec{\xi}(\omega))^{-1}=c_{0}(\vec{x})+\sum_{r=1}^{m} \sqrt{\lambda_{r}} c_{r}(\vec{x}) \xi_{r}(\omega),
$$

where $\xi_{r}, r=1, \ldots, m$, are assumed to be continuous, independent, and identically distributed random variables, and $\left(\lambda_{r}, c_{r}\right)$ are acquired by solving an eigenvalue problem in the form of a Fredholm integral equation as discussed, e.g., in [12. Introducing the random vector $\vec{\xi}=\left(\xi_{1}, \ldots, \xi_{m}\right)$ and assuming that $f=f(\vec{x}, \vec{\xi})$, we then define $\mathcal{F}=\sigma(\vec{\xi})$, which is understood to be the minimal $\sigma$-algebra generated by $\vec{\xi}$. With the problem defined in this manner, then providing random fields $p$ and $\vec{u}$ can be found that satisfy (2), the Doob-Dynkin lemma, which is given, e.g., in [5], tells us that they will be expressible as Borel functions of $\vec{\xi}$ for each $\vec{x} \in D$, i.e. $p=p(\vec{x}, \vec{\xi})$ and $\vec{u}=\vec{u}(\vec{x}, \vec{\xi})$.

Now note, that given $\xi_{r}(\Omega)=\Gamma, r=1, \ldots, m$, and denoting the density function of $\xi_{r}$ by $\rho_{r}$, we have, for any Borel function $g=g(\vec{y})$,

$$
\int_{\Omega} g(\vec{\xi}(\omega)) d P=\int_{\Gamma^{m}} g(\vec{y}) \rho(\vec{y}) d \vec{y}
$$

where $\rho(\vec{y})=\rho_{1}\left(y_{1}\right) \cdots \rho_{m}\left(y_{m}\right)$ and $d \vec{y}=d y_{1} \cdots d y_{m}$. Here $\rho$ is the joint density function of $\vec{\xi}$, and the above integrals represent the expected value of $g(\vec{\xi})$. As all the random fields in this paper are Borel functions of $\vec{\xi}$ for each $\vec{x} \in D$, (4) can be used to evaluate all integrals over the sample space, $\Omega$, that appear in the sequel.

As mentioned above, the expression for $c^{-1}$ given by (3) is derived from curtailing an infinite Karhunen-Loève expansion, retaining only the $m$ most significant terms (cf. [12] and [2]). The number of terms required, $m$, is computed a priori, usually by considering the decay of the eigenvalues, $\left(\lambda_{r}\right)$, of the covariance function of $c^{-1}$ (see [10] for a discussion). Ideally, $m$ should be chosen so that the truncation error and the numerical discretization errors (to be introduced) are balanced. We will assume that $m$ is chosen large enough so that the reciprocal of the diffusion coefficient (and hence the diffusion coefficient itself) is bounded between two positive constants 
independent of $m$ almost everywhere, i.e.

$$
a_{\min } \leq c(\vec{x}, \vec{\xi}(\omega))^{-1} \leq a_{\max } \quad \forall \vec{x} \in D \quad P \text {-a.e. }
$$

where by $P$-a.e. we mean that there exists a set $E \in \mathcal{F}$ with $P(E)=0$ such that the proposition holds on the complement of $E$. Under these assumptions, the convergence rate of the proposed algorithm will be shown to be independent of $m$ but, as is usual for diffusion problems, not of $a_{\min }$ and $a_{\max }$. Numerical results will be given in 88 to support this conclusion. Note that (5) will not hold in general for arbitrary distributions of the random variables and arbitrary covariance functions. Sufficient conditions are discussed in [10. In particular, it is necessary that the random variables be bounded.

2.2. Function space notation. We will make use of the Lebesgue spaces $L_{2}$ and $L_{\infty}$ on $D$ and $\Omega$, and the Sobolev spaces $H^{m}$, where $m$ is an integer, on $D$. These spaces and their associated inner products and norms are defined in numerous texts, e.g., [11. Note that when $m=0, H^{m}(D)=L_{2}(D)$.

The spaces $H^{m}(D)^{2}$ are understood to contain vector fields whose components are in $H^{m}(D)$, i.e. given $\vec{v}=\left(v_{1}, v_{2}\right) \in H^{m}(D)^{2}$, then $v_{1}, v_{2} \in H^{m}(D)$. Inner products on these vector function spaces are defined by summing the inner products on the components, i.e.

$$
(\vec{v}, \vec{w})_{H^{m}(D)^{2}}=\left(v_{1}, w_{1}\right)_{H^{m}(D)}+\left(v_{2}, w_{2}\right)_{H^{m}(D)},
$$

with the induced norms following. The space $H(\operatorname{div} ; D)$ is understood to be the space of functions in $L_{2}(D)^{2}$ whose divergence is in $L_{2}(D)$. It has the inner product

$$
(\vec{v}, \vec{w})_{H(\operatorname{div} ; D)}=(\vec{v}, \vec{w})_{L_{2}(D)^{2}}+(\nabla \cdot \vec{v}, \nabla \cdot \vec{w})_{L_{2}(D)}
$$

from which the norm $\|\cdot\|_{H(\operatorname{div} ; D)}$ is induced.

The random fields in this paper will live in tensor products of spaces defined on the spatial domain, $D$, with spaces defined on the sample space, $\Omega$. The space $H^{m}(D) \otimes L_{2}(\Omega)$ is given by

$$
H^{m}(D) \otimes L_{2}(\Omega)=\left\{v: D \times \Omega \rightarrow \mathbb{R}\left|D^{\gamma} v \in L_{2}(D) \otimes L_{2}(\Omega),\right| \gamma \mid \leq m\right\},
$$

with inner product

$$
(v, w)_{H^{m}(D) \otimes L_{2}(\Omega)}=\sum_{|\gamma| \leq m} \int_{\Omega} \int_{D} D^{\gamma} v D^{\gamma} w,
$$

where $D^{\gamma} v$ represents weak derivatives of $v$, i.e.

$$
D^{\gamma} v=\frac{\partial^{|\gamma|} v}{\partial x_{1}^{\gamma_{1}} \partial x_{2}^{\gamma_{2}}}
$$

with $\gamma=\left(\gamma_{1}, \gamma_{2}\right) \in \mathbb{N}^{2}$ and $|\gamma|=\gamma_{1}+\gamma_{2}$. This inner product induces the norm $\|\cdot\|_{H^{m}(D) \otimes L_{2}(\Omega)}$. The spaces $H^{m}(D)^{2} \otimes L_{2}(\Omega)$ and $H(\operatorname{div} ; D) \otimes L_{2}(\Omega)$ are defined in an analogous fashion. Tensor products of Hilbert spaces are discussed more formally in [2] and [22].

The space $L_{\infty}(D) \otimes L_{\infty}(\Omega)$ is the space of all random fields on $D \times \Omega$ whose absolute value has a finite essential supremum. The norm associated with this space is defined via this essential supremum, i.e.

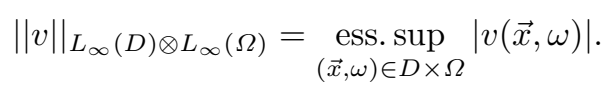


From this definition, we see that $\left\|c^{-1}\right\|_{L_{\infty}(D) \otimes L_{\infty}(\Omega)} \leq a_{\max }$, where $a_{\max }$ is as in (5).

2.3. Weighted $H($ div $; D) \otimes L_{2}(\Omega)$ bilinear form. We define the Hilbert space $U$ to consist of the vector space $L_{2}(D)^{2} \otimes L_{2}(\Omega)$ paired with the inner product

$$
(\vec{v}, \vec{w})_{U}=\int_{\Omega} \int_{D} \frac{1}{c} \vec{v} \cdot \vec{w}
$$

This induces the norm $\|\vec{v}\|_{U}$.

We define a weighted $H(\operatorname{div} ; D) \otimes L_{2}(\Omega)$ bilinear form $\Lambda: H(\operatorname{div} ; D) \otimes L_{2}(\Omega) \times$ $H(\operatorname{div} ; D) \otimes L_{2}(\Omega) \rightarrow \mathbb{R}$ by

$$
\Lambda(\vec{v}, \vec{w})=(\vec{v}, \vec{w})_{U}+(\nabla \cdot \vec{v}, \nabla \cdot \vec{w})_{L_{2}(\Omega) \otimes L_{2}(D)} .
$$

This differs from the standard $H($ div $D) \otimes L_{2}(\Omega)$ bilinear form only by the weight $c^{-1}$ in the first term. As $c^{-1}$ is bounded almost everywhere between two positive constants, as noted in ( $(\underline{5)}), \Lambda(\cdot, \cdot)$ induces a norm, to be denoted as $\|\cdot\|_{\Lambda}$. Note that this norm is equivalent to $\|\cdot\|_{H(\text { div } ; D) \otimes L_{2}(\Omega)}$.

2.4. Weak formulation. Let $c \in L_{\infty}(D) \otimes L_{\infty}(\Omega)$ and $f \in L_{2}(D) \otimes L_{2}(\Omega)$. The weak formulation of the first-order boundary value problem given in $\$ 2.1$ is: find $\vec{u} \in H(\operatorname{div} ; D) \otimes L_{2}(\Omega)$ and $p \in L_{2}(D) \otimes L_{2}(\Omega)$ such that

$$
\begin{aligned}
a(\vec{u}, \vec{v})+b(p, \vec{v}) & =0 & & \forall \vec{v} \in H(\operatorname{div} ; D) \otimes L_{2}(\Omega), \\
b(q, \vec{u}) & =-l(q) & & \forall q \in L_{2}(D) \otimes L_{2}(\Omega),
\end{aligned}
$$

where

$$
a(\vec{v}, \vec{w})=(\vec{v}, \vec{w})_{U}, \quad b(q, \vec{v})=(q, \nabla \cdot \vec{v})_{L_{2}(D) \otimes L_{2}(\Omega)}, \quad l(q)=(f, q)_{L_{2}(D) \otimes L_{2}(\Omega)} .
$$

This will possess a unique solution, as shown in [4, provided that the bilinear forms $a(\cdot, \cdot)$ and $b(\cdot, \cdot)$ are continuous, $a(\cdot, \cdot)$ is coercive on the null-space of $b(\cdot, \cdot)$, and that there exists a constant $\beta>0$, called the inf-sup constant, such that

$$
\sup _{\substack{\vec{v} \in H(\operatorname{div} ; D) \otimes \\ L_{2}(\Omega) \backslash\{0\}}} \frac{b(q, \vec{v})}{\|\vec{v}\|_{H(\operatorname{div} ; D) \otimes L_{2}(\Omega)}} \geq \beta\|q\|_{L_{2}(D) \otimes L_{2}(\Omega)} \quad \forall q \in L_{2}(D) \otimes L_{2}(\Omega) .
$$

The continuity and coercivity conditions on $a(\cdot, \cdot)$ and $b(\cdot, \cdot)$ are readily demonstrated, with the conditions on $a(\cdot, \cdot)$ being satisfied as a consequence of (5). The existence of $\beta$ was established in [9], where, moreover, it was shown to depend only on the spatial domain $D$ and to be equal to its counterpart in the analogous deterministic analysis.

2.5. Polynomial chaos. The polynomial chaos expansion method, as pioneered in [12] and generalized in [23], consists of constructing a finite-dimensional subspace of $L_{2}(\Omega)$, here denoted by $T$, that is the span of the $m$-variate polynomials from the Askey scheme of hypergeometric polynomials (discussed in [20]) that are orthogonal with respect to the underlying probability measure. That is, $T=\operatorname{span}\left\{\chi_{1}, \ldots, \chi_{M}\right\}$, where

$$
\int_{\Omega} \chi_{k}(\vec{\xi}) \chi_{l}(\vec{\xi}) d P=\int_{\Gamma^{m}} \chi_{k}(\vec{y}) \chi_{l}(\vec{y}) \rho(\vec{y}) d y=\kappa_{k} \delta_{k l}
$$

and

$$
M=\frac{(m+n) !}{m ! n !}
$$


Note that if $\rho$ is the density function corresponding to an $m$-dimensional Gaussian distribution, then the polynomial chaos will consist of $m$-variate Hermite polynomials. If $\rho$ is the density function corresponding to an $m$-dimensional uniform distribution, then the polynomial chaos will consist of $m$-variate Legendre polynomials. For polynomials corresponding to other distributions, see 24].

2.6. Finite element formulation. Let $\mathcal{T}=\left\{\triangle_{1}, \ldots, \triangle_{K}\right\}$ be a triangulation on $D$. We assume that the triangulation belongs to a family of triangulations that are quasi-uniform and shape regular. Let $h_{k}$ be the length of the longest side of $\triangle_{k}$ and define $h=\max h_{k}$ to be the mesh parameter of $\mathcal{T}$.

In the following we will use tensor products of finite-dimensional vector spaces. Note that such spaces are spanned by products of basis functions from the vector spaces that comprise the tensor product. The zeroth-order Raviart-Thomas space is defined by

$$
R=\left\{\vec{v} \in H(\operatorname{div} ; D)|v|_{\triangle_{k}} \in P_{0}\left(\triangle_{k}\right)^{2}+P_{0}\left(\triangle_{k}\right) \vec{x}\right\},
$$

where $P_{0}\left(\triangle_{k}\right)$ denotes polynomials of zero degree, i.e. constants on $\triangle_{k}$. Let $\left\{\vec{\phi}_{1}, \ldots, \vec{\phi}_{N_{R}}\right\}$ be a basis of $R$ and let $Q=\operatorname{span}\left\{\psi_{1}, \ldots, \psi_{N_{Q}}\right\}$ be the set of piecewise constants on the triangulation $\mathcal{T}$. Then $R \otimes T \subset H(\operatorname{div} ; D) \otimes L_{2}(\Omega)$ and $Q \otimes T \subset L_{2}(D) \otimes L_{2}(\Omega)$. Note that $R$ and $Q$ are known to satisfy a discrete deterministic inf-sup condition, as shown, for example, in [4] and [19.

The finite element formulation of the weak formulation given in $\$ 2.4$ is given by: find $\vec{u}_{h n} \in R \otimes T$ and $p_{h n} \in Q \otimes T$ such that

$$
\begin{aligned}
a\left(\vec{u}_{h n}, \vec{v}\right)+b\left(p_{h n}, \vec{v}\right) & =0 & & \forall \vec{v} \in R \otimes T, \\
b\left(q, \vec{u}_{h n}\right) & =-l(q), & & \forall q \in Q \otimes T .
\end{aligned}
$$

This possesses a unique solution under the same conditions that apply for the weak formulation. The discrete continuity and coercivity conditions on $a(\cdot, \cdot)$ and $b(\cdot, \cdot)$ follow in an analogous way to the infinite-dimensional case considered in $\$ 2.4$. Existence and uniqueness are then assured if it can be shown that there exists a constant $\beta_{h n}>0$ such that

$$
\sup _{\vec{v} \in R \otimes T \backslash\{0\}} \frac{b(q, \vec{v})}{\|\vec{v}\|_{H(\operatorname{div} ; D) \otimes L_{2}(\Omega)}} \geq \beta_{h n}\|q\|_{L_{2}(D) \otimes L_{2}(\Omega)} \quad \forall q \in Q \otimes T .
$$

The existence of $\beta_{h n}$ can be demonstrated by employing Fortin's Lemma (see [3]) as follows. First we note that by considering $\nabla \cdot \nabla s=w$, where $w \in L_{2}(D) \otimes T$, it can be shown that there exists a unique $\vec{z} \in H^{1}(D)^{2} \otimes T$ such that

$$
\|\vec{z}\|_{H(\operatorname{div} ; D) \otimes L_{2}(\Omega)} \leq C_{D}\|w\|_{L_{2}(D) \otimes L_{2}(\Omega)},
$$

where $C_{D}$ is only dependent on the spatial domain. Using standard arguments it then follows that the constant $\beta>0$ satisfying (7) also satisfies a semi-discrete inf-sup condition given by

$$
\sup _{\vec{v} \in H^{1}(D)^{2} \otimes T \backslash\{0\}} \frac{b(q, \vec{v})}{\|\vec{v}\|_{H(\operatorname{div} ; D) \otimes L_{2}(\Omega)}} \geq \beta\|q\|_{L_{2}(D) \otimes L_{2}(\Omega)} \quad \forall q \in L_{2}(D) \otimes T .
$$

Now suppose there exists an operator $\Pi: H^{1}(D)^{2} \otimes T \rightarrow R \otimes T$ such that for each $\vec{v} \in H^{1}(D) \otimes T$

$$
\begin{aligned}
b(\Pi \vec{v}-\vec{v}, q) & =0 \quad \forall q \in L_{2}(D) \otimes L_{2}(\Omega), \\
\|\left.\Pi \vec{v}\right|_{H(\operatorname{div} ; D) \otimes L_{2}(\Omega)} & \leq C_{\Pi}\|\vec{v}\|_{H(\operatorname{div} ; D) \otimes L_{2}(\Omega)},
\end{aligned}
$$


where $C_{\Pi}$ is independent of $h, m$, and $n$. Then

$$
\begin{aligned}
\sup _{\vec{v} \in R \otimes T \backslash\{0\}} \frac{b(q, \vec{v})}{\|\vec{v}\|_{H(\operatorname{div} ; D) \otimes L_{2}(\Omega)}} & =\sup _{\vec{v} \in H^{1}(D)^{2} \otimes T \backslash\{0\}} \frac{b(q, \Pi \vec{v})}{\|\Pi \vec{v}\|_{H(\operatorname{div} ; D) \otimes L_{2}(\Omega)}} \\
& =\sup _{\vec{v} \in H^{1}(D)^{2} \otimes T \backslash\{0\}} \frac{b(q, \Pi \vec{v}-\vec{v})+b(\vec{v}, q)}{\|\Pi \vec{v}\|_{H(\operatorname{div} ; D) \otimes L_{2}(\Omega)}} \\
& \geq \frac{\beta}{C_{\Pi}}\|q\|_{L_{2}(D) \otimes L_{2}(\Omega) \quad \forall q \in Q \otimes T .}
\end{aligned}
$$

An operator $\Pi$ satisfying the above properties is given by the Raviart-Thomas interpolation operator described in $\$ 2.8$. Therefore, the existence of the discrete inf-sup constant is established and, moreover, it is independent of the discretization parameters $h, m$, and $n$.

In [6], the well-posedness of a stochastic saddle-point problem arising in the solution of a PDE with stochastic boundary was analyzed. In that work, an inf-sup stability result was established under the assumption that a particular discrete space is chosen. Here, we note that (9) holds independently of the choice of $T \subset L_{2}(\Omega)$.

2.7. Matrix formulation. The finite element formulation given in \$2.6 can be written as a matrix problem. This is obtained by using the expansions

$$
\vec{u}_{h n}=\sum_{j=1}^{N_{R}} \sum_{l=1}^{M} u_{j l} \vec{\phi}_{j} \chi_{l}, \quad \quad p_{h n}=\sum_{j=1}^{N_{Q}} \sum_{l=1}^{M} p_{j l} \psi_{j} \chi_{l}
$$

and allowing $\vec{v}$ to vary over the basis functions of $R \otimes T$ and $q$ to vary over the basis functions of $Q \otimes T$. This leads to a matrix problem of the form

$$
\left[\begin{array}{cc}
A & B^{T} \\
B & 0
\end{array}\right]\left[\begin{array}{l}
\mathbf{u} \\
\mathbf{p}
\end{array}\right]=\left[\begin{array}{l}
\mathbf{0} \\
\mathbf{f}
\end{array}\right] .
$$

The matrices that appear in the system matrix can be decomposed as

$$
A=G_{0} \otimes K_{0}+\sum_{r=1}^{m} \sqrt{\lambda_{r}} G_{r} \otimes K_{r}, \quad B=G_{0} \otimes B_{0},
$$

with entries given by

$$
\begin{aligned}
& {\left[G_{r}\right]_{k l}=\int_{\Omega} \xi_{r} \chi_{k} \chi_{l} \quad\left(=\int_{\Gamma^{m}} y_{r} \chi_{k} \chi_{l} \rho\right)} \\
& {\left[K_{r}\right]_{i j}=\int_{D} c_{r} \vec{\phi}_{i} \cdot \vec{\phi}_{j}, \quad\left[B_{0}\right]_{i j}=\int_{D} \psi_{i} \nabla \cdot \vec{\phi}_{j},}
\end{aligned}
$$

where $\xi_{0}=1$ in the definition of $G_{0}$. The vector $\mathbf{f}$ has entries given by

$$
\mathbf{f}=\left[\begin{array}{c}
\mathbf{f}_{1} \\
\vdots \\
\mathbf{f}_{M}
\end{array}\right], \quad\left[\mathbf{f}_{k}\right]_{i}=-\int_{\Omega} \int_{D} f \psi_{i} \chi_{k} .
$$

The solution vector contains the coefficients of $\vec{u}_{h n}$ and $p_{h n}$ stacked columnwise. Once it has been computed, we have approximations to the random fields $\vec{u}$ and $p$ that satisfy the weak formulation of the diffusion problem. These approximations can then be post-processed to obtain probabilistic information about the solution variables. The means and variances can be trivially computed, and the probability distributions of quantities of interest can be computed via sampling of $\vec{\xi}$. 
2.8. Interpolation operator. We here define a spatial interpolation operator $\Pi: H^{1}(D)^{2} \otimes L_{2}(\Omega) \rightarrow R \otimes L_{2}(\Omega)$ such that for each $\omega \in \Omega, \Pi \operatorname{maps} v(\vec{x}, \vec{\xi})$ to a function in $R$ in the conventional manner (see, e.g., [3, p. 147]); i.e. , $\Pi$ acts only on the spatial components of the random fields in its domain and does so in a manner analogous to its deterministic counterpart. To be more precise, we first define $\Pi_{k}: H^{1}\left(\triangle_{k}\right)^{2} \otimes L_{2}(\Omega) \rightarrow R \otimes L_{2}(\Omega)$ such that for $\vec{v} \in H^{1}\left(\triangle_{k}\right)^{2} \otimes L_{2}(\Omega)$,

$$
\int_{e_{i}}\left(\vec{v}-\Pi_{k} \vec{v}\right) \cdot \vec{n}_{i}=0, \quad i=1,2,3, \quad \forall \omega \in \Omega,
$$

where $e_{i}, i=1,2,3$, are the edges of $\triangle_{k}$, and $\vec{n}_{i}, i=1,2,3$, are the respective unit normal vectors to these edges. Then $\Pi$ is defined such that for $\vec{v} \in H^{1}(D)^{2} \otimes L_{2}(\Omega)$,

$$
\left.(\Pi \vec{v})\right|_{\triangle_{k}}=\Pi_{k}\left(\left.\vec{v}\right|_{\triangle_{k}}\right)
$$

Following [1], we have the approximation property

$$
\|\vec{v}-\Pi \vec{v}\|_{L_{2}(D)^{2}} \leq C_{0} h\|\vec{v}\|_{H^{1}(D)^{2}} \quad \forall \omega \in \Omega,
$$

where $C_{0}$ is a constant. Squaring and integrating over $\Omega$ gives

$$
\|\vec{v}-\Pi \vec{v}\|_{L_{2}(D)^{2} \otimes L_{2}(\Omega)} \leq C_{0} h\|\vec{v}\|_{H^{1}(D)^{2} \otimes L_{2}(\Omega)} .
$$

2.9. Semi-discrete formulation. We here introduce a semi-discrete formulation of the weak formulation of the diffusion problem where only the space $T \subset L_{2}(\Omega)$ is finite-dimensional. This is given by: find $\vec{u}_{n} \in H(\operatorname{div} ; D) \otimes T$ and $p_{n} \in L_{2}(D) \otimes T$ such that

$$
\begin{aligned}
a\left(\vec{u}_{n}, \vec{v}\right)+b\left(p_{n}, \nabla \cdot \vec{v}\right) & =0 & & \forall v \in H(\operatorname{div} ; D) \otimes T, \\
b\left(q, \nabla \cdot \vec{u}_{n}\right) & =-l(q) & & \forall q \in L_{2}(D) \otimes T .
\end{aligned}
$$

This possesses a unique solution under an analogous set of conditions as for the weak formulation and the finite element formulation discussed in $\$ 2.4$ and $\$ 2.6$. respectively. In particular, the inf-sup condition that is required, over the relevant semi-discrete space, was established in \$2.6 and is given by (10).

In order to obtain a bound on the error between the flux solutions to the semidiscrete and the fully discrete problems we make the following assumption. Given $f \in L_{2}(D) \otimes T$ we assume that $\vec{u}_{n} \in H^{1}(D)^{2} \otimes T$ and that for each realization of $\vec{\xi}$,

$$
\left\|\vec{u}_{n}\right\|_{H^{1}(D)^{2}} \leq C_{r}\|f\|_{L_{2}(D)}
$$

from which it follows that

$$
\left\|\vec{u}_{n}\right\|_{H^{1}(D)^{2} \otimes L_{2}(\Omega)} \leq C_{r}\|f\|_{L_{2}(D) \otimes L_{2}(\Omega)} .
$$

Theorem 1. Given $f \in L_{2}(D) \otimes T$, then the flux solution to the semi-discrete problem, $\vec{u}_{n}$, and the flux solution to the fully discrete problem, $\vec{u}_{h n}$, satisfy

$$
\left\|\vec{u}_{n}-\vec{u}_{h n}\right\|_{U} \leq C_{*} h\|f\|_{L_{2}(D) \otimes L_{2}(\Omega)},
$$

where $C_{*}$ is independent of $h, m$, and $n$, and $a_{\max }$ is as in (5).

Proof. Using the upper bound on $c^{-1}$ in (5) and following [4] we have

$$
\begin{aligned}
\left\|\vec{u}_{n}-\vec{u}_{h n}\right\|_{U} & \leq a_{\max }^{1 / 2}\left\|\vec{u}_{n}-\vec{u}_{h n}\right\|_{L_{2}(D) \otimes L_{2}(\Omega)} \\
& \leq a_{\max }^{1 / 2} C_{\star} \inf _{\vec{v} \in R \otimes T}\left\|\vec{u}_{n}-\vec{v}\right\|_{L_{2}(D) \otimes L_{2}(\Omega)},
\end{aligned}
$$


where $C_{\star}$ is a constant. Now, as $\Pi \vec{u}_{n} \in R \otimes T$, using (13) and (14) we have

$$
\begin{aligned}
\left\|\vec{u}_{n}-\vec{u}_{h n}\right\|_{U} & \leq a_{\max }^{1 / 2} C_{\star} C_{0} h\left\|\vec{u}_{n}\right\|_{H^{1}(D)^{2} \otimes L_{2}(\Omega)} \\
& \leq a_{\max }^{1 / 2} C_{\star} C_{0} C_{r} h\|f\|_{L_{2}(D) \otimes \| L_{2}(\Omega)},
\end{aligned}
$$

which demonstrates the theorem with $C_{*}=a_{\max }^{1 / 2} C_{\star} C_{0} C_{r}$.

\section{Helmholtz decomposition, projection and $H($ div; $D$ ) operators}

In this section we describe a Helmholtz decomposition of $\vec{v} \in R \otimes T$ analogous to its deterministic counterpart given in [1]. We also introduce some projection operators and a weighted $H(\operatorname{div} ; D) \otimes L_{2}(\Omega)$ operator which, along with the Helmholtz decomposition, will be used in $\S \$ 5$, 6 and 7 .

3.1. Helmholtz decomposition. Let $W=\operatorname{span}\left\{\varphi_{1}, \ldots, \varphi_{N_{W}}\right\}$ be the set of piecewise linear functions defined on the triangulation $\mathcal{T}$. Here $N_{W}$ will equal the number of nodes in the triangulation. It is well known, e.g., see [1], that

$$
\{\vec{v} \in R \mid \nabla \cdot \vec{v}=0\}=\{\nabla \times w \mid w \in W\} .
$$

As divergence and curl are purely spatial operators, i.e. they don't affect the stochastic nature of the random fields they act upon, it follows that

$$
\{\vec{v} \in R \otimes T \mid \nabla \cdot \vec{v}=0\}=\{\nabla \times w \mid w \in W \otimes T\} .
$$

Here $\nabla \times w=\left(-\partial w / \partial x_{2}, \partial w / \partial x_{1}\right)$.

We now define a weighted discrete gradient operator (cf. [1, eq. (3.1)]), denoted $\operatorname{grad}_{h}^{c}: Q \otimes T \rightarrow R \otimes T$, such that for $q \in Q \otimes T$,

$$
\left(\operatorname{grad}_{h}^{c} q, \vec{v}\right)_{U}=-(q, \nabla \cdot \vec{v})_{L_{2}(D) \otimes L_{2}(\Omega)} \quad \forall \vec{v} \in R \otimes T .
$$

The superscript $c$ denotes the dependence of the operator on the random field $c$ through the definition of $(\cdot, \cdot)_{U}$.

Theorem 2 (Helmholtz Decomposition). Given $\vec{v} \in R \otimes T$, there exists $q \in Q \otimes T$ and $w \in W \otimes T$ such that

$$
\vec{v}=\operatorname{grad}_{h}^{c} q+\nabla \times w .
$$

Proof. To establish the given decomposition, it is sufficient to show that $\operatorname{grad}_{h}^{c}(Q \otimes T)$ and $\nabla \cdot(W \otimes T)$ are orthogonal complements on $R \otimes T$ with respect to $(\cdot, \cdot)_{U}$. To see this, let $w \in W \otimes T$; then

$$
\begin{aligned}
\nabla \cdot(\nabla \times w)=0 & \Leftrightarrow(\nabla \cdot(\nabla \times w), q)_{L_{2}(D) \otimes L_{2}(\Omega)}=0 & & \forall q \in Q \otimes T \\
& \Leftrightarrow\left(\nabla \times w, \operatorname{grad}_{h}^{c} q\right)_{U}=0 & & \forall q \in Q \otimes T \\
& \left.\Leftrightarrow \nabla \times w \in \operatorname{(grad}_{h}^{c}(Q \otimes T)\right)^{\perp} . & &
\end{aligned}
$$

Note that, for $q \in Q \otimes T$ and $w \in W \otimes T, \operatorname{grad}_{h}^{c} q$ and $\nabla \times w$ are also orthogonal with respect to $(\cdot, \cdot)_{\Lambda}$, where $(\cdot, \cdot)_{\Lambda}$ is as defined in $\$ 2.3$. 
3.2. Projection operators. We define the projection operator $\Theta: L_{2}(D) \otimes L_{2}(\Omega)$ $\rightarrow Q \otimes T$ such that for each $v \in L_{2}(D) \otimes L_{2}(\Omega)$,

$$
(\Theta v, q)_{L_{2}(D) \otimes L_{2}(\Omega)}=(v, q)_{L_{2}(D) \otimes L_{2}(\Omega)} \quad \forall q \in Q \otimes T .
$$

The operator $\Theta$ affects both the spatial and the stochastic aspect of the random fields on which it operates. It will be in our interest to define an operator that only affects the spatial aspect of the random fields it acts upon. Therefore, we define $\Sigma: L_{2}(D) \otimes L_{2}(\Omega) \rightarrow Q \otimes L_{2}(\Omega)$ such that for each $v \in L_{2}(D) \otimes L_{2}(\Omega)$,

$$
(\Sigma v, q)_{L_{2}(D)}=(v, q)_{L_{2}(D)} \quad \forall q \in Q \quad \forall \omega \in \Omega, ;
$$

that is, for each $\omega \in \Omega, \Sigma$ is the $L_{2}(D)$-projection of $v(\vec{x}, \vec{\xi})$ onto $Q$. It can then be shown that given $\vec{v} \in H^{1}(D)^{2} \otimes L_{2}(\Omega)$ we have the commutativity property

$$
\nabla \cdot \Pi \vec{v}=\Sigma \nabla \cdot \vec{v}, \quad \forall \omega \in \Omega,
$$

the proof of which follows that given in [3] for the deterministic case. Using this, we also have for $\vec{v} \in R \otimes T$ and $q \in Q \otimes T$,

$$
\begin{aligned}
(\Theta \nabla \cdot \vec{v}, q)_{L_{2}(D) \otimes L_{2}(\Omega)} & =\int_{\Omega}(\nabla \cdot \vec{v}, q)_{L_{2}(D)}=\int_{\Omega}(\Sigma \nabla \cdot \vec{v}, q)_{L_{2}(D)} \\
& =\int_{\Omega}(\nabla \cdot \Pi \vec{v}, q)_{L_{2}(D)}=(\nabla \cdot \Pi \vec{v}, q)_{L_{2}(D) \otimes L_{2}(\Omega)},
\end{aligned}
$$

which is analogous to the deterministic result given in 1 .

We define $\mathcal{P}: H(\operatorname{div} ; D) \otimes L_{2}(\Omega) \rightarrow R \otimes T$ such that for each $\vec{w} \in H(\operatorname{div} ; D) \otimes$ $L_{2}(\Omega)$,

$$
\Lambda(\mathcal{P} \vec{w}, \vec{v})=\Lambda(\vec{w}, \vec{v}) \quad \forall \vec{v} \in R \otimes T .
$$

That is, $\mathcal{P}$ is the projection operator onto $R \otimes T$ defined with respect to $\Lambda(\cdot, \cdot)$.

3.3. Weighted $H(\operatorname{div} ; D) \otimes L_{2}(\Omega)$ operator. We next define the weighted $H(\operatorname{div} ; D) \otimes L_{2}(\Omega)$ operator $\mathcal{H}: R \otimes T \rightarrow R \otimes T$ such that for each $\vec{w} \in R \otimes T$,

$$
(\mathcal{H} \vec{w}, \vec{v})_{U}=\Lambda(\vec{w}, \vec{v}) \quad \forall \vec{v} \in R \otimes T .
$$

Note that $\mathcal{H}$ is a positive-definite symmetric operator. Therefore we can define its inverse $\mathcal{H}^{-1}: R \otimes T \rightarrow R \otimes T$ such that for each $\vec{w} \in R \otimes T$,

$$
\Lambda\left(\mathcal{H}^{-1} \vec{w}, v\right)=(\vec{w}, \vec{v})_{U} \quad \forall \vec{v} \in R \otimes T .
$$

Note also that $\mathcal{H}$ maps $\operatorname{grad}_{h}^{c}(Q \otimes T)$ onto itself. To see this, let $\vec{w}=\operatorname{grad}_{h}^{c} q$ for some $q \in Q \otimes T$. Then, using (6) and (16),

$$
\begin{aligned}
(\mathcal{H} \vec{w}, \vec{v})_{U} & =\left(\operatorname{grad}_{h}^{c} q, \vec{v}\right)_{U}+\left(\nabla \cdot \operatorname{grad}_{h}^{c} q, \nabla \cdot \vec{v}\right)_{L_{2}(D) \otimes L_{2}(\Omega)} \\
& =\left(\operatorname{grad}_{h}^{c} q, \vec{v}\right)_{U}-\left(\operatorname{grad}_{h}^{c} \nabla \cdot \operatorname{grad}_{h}^{c} q, \vec{v}\right)_{U} \\
& =\left(\operatorname{grad}_{h}^{c}\left(q-\nabla \cdot \operatorname{grad}_{h}^{c} q\right), \vec{v}\right)_{U} \quad \forall \vec{v} \in R \otimes T .
\end{aligned}
$$

As $q-\nabla \cdot \operatorname{grad}_{h}^{c} q \in Q \otimes T$ it follows that given $q \in Q \otimes T$ there exists $q^{\prime} \in Q \otimes T$ such that $\mathcal{H g r a d}_{h}^{c} q=\operatorname{grad}_{h}^{c} q^{\prime}$. Similarly, for $q \in Q \otimes T$, it can be shown that there exists $q^{\prime} \in Q \otimes T$ such that $\mathcal{H}^{-1} \operatorname{grad}_{h}^{c} q=\operatorname{grad}_{h}^{c} q^{\prime}$. 


\section{SOlVING THE FIRST-ORDER DIFFUSION PROBLEM}

The coefficient matrix given in (12), denoted here by $C$, is sparse, symmetric, and indefinite. Hence, a suitable solution scheme is the MINRES algorithm, described e.g., in [16]. This is a Krylov subspace method that minimizes the Euclidean norm of the residual error at each step. In order to ensure that the iteration is efficient, preconditioning is essential. Ideally, we want the number of MINRES iterations required to reduce the error to within a specified tolerance to be independent of all the discretization parameters, and so we seek a preconditioner $P$ such that the eigenvalues of $P^{-1} C$ are independent of the discretization parameters $h, n$, and $m$.

4.1. Deterministic $H(\operatorname{div} ; D)$ preconditioning. Recall that in the deterministic diffusion problem, where the diffusion coefficient, $c=c_{0}(\vec{x})$, and the source function, $f=f_{0}(\vec{x})$, are known (deterministic) fields, the standard mixed Galerkin discretization gives rise to a linear system of the form

$$
\left[\begin{array}{cc}
K_{0} & B_{0}^{T} \\
B_{0} & 0
\end{array}\right]\left[\begin{array}{l}
\mathbf{u}_{0} \\
\mathbf{p}_{0}
\end{array}\right]=\left[\begin{array}{c}
\mathbf{0} \\
\mathbf{f}_{0}
\end{array}\right]
$$

where $B_{0} \in \mathbb{R}^{N_{Q} \times N_{R}}$ and $K_{0} \in \mathbb{R}^{N_{R} \times N_{R}}$ are defined as in 2.7 . The so-called ideal $H(\operatorname{div} ; D)$ preconditioner given by

$$
P_{0}=\left[\begin{array}{cc}
K_{0}+D_{0} & 0 \\
0 & N_{0}
\end{array}\right]
$$

where $D_{0} \in \mathbb{R}^{N_{R} \times N_{R}}$ and $N_{0} \in \mathbb{R}^{N_{Q} \times N_{Q}}$ are defined via

$$
\left[D_{0}\right]_{i j}=\int_{D}\left(\nabla \cdot \vec{\phi}_{i}\right)\left(\nabla \cdot \vec{\phi}_{j}\right), \quad\left[N_{0}\right]_{i j}=\int_{D} \psi_{i} \psi_{j},
$$

was proposed in [1] for the case $c_{0}(\vec{x})=1$ and subsequently analyzed in [18] and [17] for arbitrary diffusion coefficients. This choice of preconditioner is motivated by the observation that $\forall \vec{v} \in R$ and $\forall q \in Q$,

$$
\left\|c^{-\frac{1}{2}} \vec{v}\right\|_{L^{2}(D)^{2}}^{2}+\|\nabla \cdot \vec{v}\|_{L^{2}(D)}^{2}=\left(\mathbf{v},\left(K_{0}+D_{0}\right) \mathbf{v}\right), \quad\|q\|_{L^{2}(D)}^{2}=\left(\mathbf{q}, N_{0} \mathbf{q}\right) .
$$

Consequently, the diagonal blocks of $P_{0}$ provide representations of a pair of norms in which the chosen mixed finite element discretization is known to be inf-sup stable (see 4, 19]). Hence, $P_{0}$ mimics the mapping properties of the underlying saddlepoint matrix. The following theorem gives bounds for the eigenvalues of $P_{0}^{-1} C_{0}$, where $C_{0}$ is the system matrix in (19).

Theorem 3. The $N_{R}+N_{Q}$ eigenvalues of

$$
\left[\begin{array}{cc}
K_{0} & B_{0}^{T} \\
B_{0} & 0
\end{array}\right]\left[\begin{array}{l}
\mathbf{v} \\
\mathbf{q}
\end{array}\right]=\nu\left[\begin{array}{cc}
K_{0}+D_{0} & 0 \\
0 & N_{0}
\end{array}\right]\left[\begin{array}{l}
\mathbf{v} \\
\mathbf{q}
\end{array}\right]
$$

lie in $\left(-1,-\mu_{0}\right] \cup\{1\}$, where $\mu_{0} \in(0,1)$ is a constant independent of $h$.

Proof. See [18.

To obtain a practical scheme we need to approximate the action of the inverse of $K_{0}+D_{0}$. Suppose then that there exists some (any) matrix $V_{0}$ satisfying,

$$
\theta_{0} \leq \frac{\left(\mathbf{v},\left(K_{0}+D_{0}\right) \mathbf{v}\right)}{\left(\mathbf{v}, V_{0} \mathbf{v}\right)} \leq \bar{\theta}_{0} \leq 1, \quad \forall \mathbf{v} \in \mathbb{R}^{N_{R} \times N_{R}} \backslash\{\mathbf{0}\}
$$


where $(\cdot, \cdot)$ indicates the Euclidean inner product. The following theorem gives bounds for the eigenvalues of the preconditioned system obtained by replacing $K_{0}+$ $D_{0}$ in (20) with $V_{0}$.

Theorem 4. The $\left(N_{R}+N_{Q}\right)$ eigenvalues of

$$
\left[\begin{array}{cc}
K_{0} & B_{0}^{T} \\
B_{0} & 0
\end{array}\right]\left[\begin{array}{l}
\mathbf{v} \\
\mathbf{q}
\end{array}\right]=\nu\left[\begin{array}{cc}
V_{0} & 0 \\
0 & N_{0}
\end{array}\right]\left[\begin{array}{l}
\mathbf{v} \\
\mathbf{q}
\end{array}\right]
$$

lie in

$$
\left(-1, \frac{1}{2}\left(\theta_{0}\left(1-\mu_{0}\right)-\sqrt{\theta_{0}^{2}\left(\mu_{0}-1\right)^{2}+4 \mu_{0} \theta_{0}}\right)\right] \cup\left[\theta_{0}, 1\right],
$$

where $\mu_{0}$ is the quantity defined in Theorem 3 and $\theta_{0}$ is the constant from (21).

Proof. See [17.

In [1, Arnold et el. demonstrated that, in the case $c_{0}(\vec{x})=1$, choosing $V_{0}$ to be the matrix that represents the inverse of a certain multigrid $\mathrm{V}$-cycle operator yields $\theta_{0}=1-\delta_{0}$ and $\bar{\theta}_{0}=1$ in (21), where $\delta_{0}$ is a constant independent of $h$. Theorem 4 then tells us that the preconditioner

$$
\left[\begin{array}{cc}
V_{0} & 0 \\
0 & N_{0}
\end{array}\right]
$$

is $h$-optimal. For a general diffusion coefficient, both $\mu_{0}$ and $\delta_{0}$ depend on $c_{0}(\vec{x})$ and in [17] it was shown that (22) is not always $c$-optimal.

4.2. Stochastic $H(\operatorname{div} ; D) \otimes L_{2}(\Omega)$ preconditioning. We base our preconditioning strategy for (12) on the deterministic preconditioner described in $\$ 4.1$ That is, we define, first, the analogous ideal preconditioner

$$
P=\left[\begin{array}{cc}
A+F & 0 \\
0 & N
\end{array}\right],
$$

where $A$ is the (1,1) block of (12), $F=G_{0} \otimes D_{0}$ and $N=G_{0} \otimes N_{0}$. Now, $\forall \vec{v} \in R \otimes T$ and $\forall q \in Q \otimes T$, the blocks of the preconditioner provide representations of the tensor product norms,

$$
\|\vec{v}\|_{\Lambda}^{2}=(\mathbf{v},(A+F) \mathbf{v}), \quad\|q\|_{L^{2}(D) \otimes L^{2}(\Omega)}^{2}=(\mathbf{q}, N \mathbf{q}) .
$$

The efficiency of this preconditioner can be analyzed in exactly the same way as in the deterministic case. Recalling that $M$ is the dimension of the space $T$ defined in $\$ 2.5$ and $N_{R}$ and $N_{Q}$ are the dimensions of the deterministic spaces $R$ and $Q$ defined in 92.6 , we have the following theorem.

Theorem 5. The $M \times\left(N_{R}+N_{Q}\right)$ eigenvalues of the generalized eigenvalue problem

$$
\left[\begin{array}{cc}
A & B^{T} \\
B & 0
\end{array}\right]\left[\begin{array}{l}
\mathbf{v} \\
\mathbf{q}
\end{array}\right]=\nu\left[\begin{array}{cc}
A+F & 0 \\
0 & N
\end{array}\right]\left[\begin{array}{l}
\mathbf{v} \\
\mathbf{q}
\end{array}\right]
$$

lie in $(-1,-\mu] \cup\{1\}$, where $\mu \in(0,1)$ is a constant independent of $h, m$, and $n$.

Proof. The proof follows that for the deterministic case and will be given here in outline. First note that since $\nabla \cdot R=Q$, we have $D_{0}=B_{0}^{T} N_{0}^{-1} B_{0}$ and

$$
\begin{aligned}
B^{T} N^{-1} B & =\left(G_{0} \otimes B_{0}\right)^{T}\left(G_{0} \otimes N_{0}\right)^{-1}\left(G_{0} \otimes B_{0}\right) \\
& =G_{0} \otimes\left(B_{0}^{T} N_{0}^{-1} B_{0}\right)=G_{0} \otimes D_{0}=F .
\end{aligned}
$$


It immediately follows that there are $M N_{R}$ eigenvalues equal to one. The remaining eigenvalues are negative and satisfy

$$
B(A+F)^{-1} B^{T} \mathbf{q}=-\nu N \mathbf{q} .
$$

Now, using (9) along with the equivalence of $\|\cdot\|_{H(\operatorname{div} ; D) \otimes L_{2}(\Omega)}$ and $\|\cdot\|_{\Lambda}$, gives

$$
\beta_{h n}\|q\|_{L_{2}(D) \otimes L_{2}(\Omega)} \leq \frac{1}{\min \left\{1, a_{\max }^{-1 / 2}\right\}} \sup _{\vec{v} \in R \otimes T \backslash\{0\}} \frac{b(q, \nabla \cdot \vec{v})}{\|\vec{v}\|_{\Lambda}} \quad \forall q \in Q \otimes T .
$$

Converting this to matrix notation one then obtains

$$
\beta_{h n}^{2} \min \left\{1, a_{\max }^{-1}\right\} \leq \frac{\left(\mathbf{q}, B(A+F)^{-1} B^{T} \mathbf{q}\right)}{(\mathbf{q}, N \mathbf{q})} \quad \forall \mathbf{q} \in \mathbb{R}^{M \times N_{Q}} .
$$

Therefore, we can choose $\mu=\beta_{h n}^{2} \min \left\{1, a_{\max }^{-1}\right\}$.

To obtain a practical preconditioning scheme, we need a computationally optimal way to approximate the inverse of $A+F$ in each MINRES iteration. If we have at our disposal a matrix $V$ that satisfies

$$
\theta \leq \frac{(\mathbf{v},(A+F) \mathbf{v})}{(\mathbf{v}, V \mathbf{v})} \leq \bar{\theta} \leq 1, \quad \forall \mathbf{v} \in \mathbb{R}^{M N_{R}} \backslash\{\mathbf{0}\},
$$

then the analogue of Theorem 4 holds. Specifically, we have the following result.

Theorem 6. The $M \times\left(N_{R}+N_{Q}\right)$ eigenvalues of

$$
\left[\begin{array}{cc}
A & B^{T} \\
B & 0
\end{array}\right]\left[\begin{array}{l}
\mathbf{v} \\
\mathbf{q}
\end{array}\right]=\nu\left[\begin{array}{cc}
V & 0 \\
0 & N
\end{array}\right]\left[\begin{array}{l}
\mathbf{v} \\
\mathbf{q}
\end{array}\right]
$$

lie in

$$
\left(-1, \frac{1}{2}\left(\theta(1-\mu)-\sqrt{\theta^{2}(\mu-1)^{2}+4 \mu \theta}\right)\right] \cup[\theta, 1],
$$

where $\mu$ is as in Theorem 5 and $\theta$ is the constant from (24).

Proof. Follow the proof of Theorem 4.1 in 17, replacing deterministic matrices with their larger Kronecker product counterparts.

In the next sections, we will extend the deterministic analysis presented in [1] to construct a hybrid multigrid V-cycle operator, $\mathcal{V}: R \otimes T \rightarrow R \otimes T$ whose matrix representation $V$ provides spectral equivalence bounds (24) for $A+F$ with constant $\bar{\theta}=1$ and $\theta=1-\delta$, where $\delta$ is a constant independent of $h, m$, and $n$. The efficiency of the resulting preconditioner,

$$
\left[\begin{array}{cc}
V & 0 \\
0 & N
\end{array}\right]
$$

is then determined by the spectral inclusion bounds in Theorem 6 .

\section{TWO-GRID RESULTS}

In the following, two triangulations $\mathcal{T}_{h}$ and $\mathcal{T}_{H}$ on the spatial domain are used with mesh parameters $h$ and $H$, respectively, with $H>h$. Throughout the remainder of this section, the spaces and operators defined in $\$ 2$ will carry a subscript $h$ or $H$ to indicate the underlying triangulation with respect to which they are defined. 
5.1. Two-grid bounds. Here we obtain some bounds between functions defined on the coarse grid and related functions defined on the fine grid. The bounds are analogous to those given in Lemma 3.1 in [1].

We first note that given an arbitrary function $q_{h} \in Q_{h} \otimes T$ and defining $\overrightarrow{v_{h}}=$ $\operatorname{grad}_{h}^{c} q_{h} \in R_{h} \otimes T$, we can uniquely define $q_{H}$ and $\vec{v}_{H}$ such that $\vec{v}_{H}=\operatorname{grad}_{H}^{c} q_{H}$ and $\nabla \cdot \vec{v}_{H}=\Theta_{H} \nabla \cdot \vec{v}_{h}$. Moreover, given $f=-\nabla \cdot \operatorname{grad}_{h}^{c} q_{h},\left(q_{h}, \vec{v}_{h}\right)$ and $\left(q_{H}, \vec{v}_{H}\right)$ will be finite element approximations to the diffusion problem given by (2), as can be demonstrated using the finite element formulation of the diffusion problem given in

Lemma 1. Given $q_{h} \in Q_{h} \otimes T$ there exists $\vec{\sigma} \in R_{h} \otimes T$ such that

$$
\nabla \cdot \vec{\sigma}=q_{h}-\Theta_{H} q_{h}, \quad\|\vec{\sigma}\|_{H^{1}(D)^{2} \otimes L_{2}(\Omega)} \leq C_{1}\left\|q_{h}-\Theta_{H} q_{h}\right\|_{L_{2}(D) \otimes L_{2}(\Omega)},
$$

where $C_{1}$ is independent of $h, m$, and $n$.

Proof. Consider the semi-discrete variational problem: find $p \in H_{0}^{1}(D) \otimes T$ such that

$$
\int_{\Omega} \int_{D} \nabla p \cdot \nabla v=\int_{\Omega} \int_{D}\left(q_{h}-\Theta_{H} q_{h}\right) v \quad \forall v \in H_{0}^{1}(D) \otimes T .
$$

This can be seen as the weak formulation of the second-order diffusion problem with source function $f=q_{h}-\Theta_{H} q_{h}$. From regularity considerations (cf. [2]) we have

$$
\|p\|_{H^{2}(D)} \leq C_{D}\left\|q_{h}-\Theta_{H} q_{h}\right\|_{L_{2}(D)} \quad \forall \omega \in \Omega,
$$

where $C_{D}$ is only dependent on the boundary of the spatial domain. From this it follows that

$$
\|p\|_{H^{2}(D) \otimes L_{2}(\Omega)} \leq C_{D}\left\|q_{h}-\Theta_{H} q_{h}\right\|_{L_{2}(D) \otimes L_{2}(\Omega)} .
$$

Since $p$ solves the diffusion problem we have

$$
-\nabla^{2} p=q_{h}-\Theta_{H} q_{h} \quad \text { a.e. }
$$

Now set $\vec{\sigma}=-\nabla p \in R_{h} \otimes T$. Then

$$
\begin{aligned}
\|\vec{\sigma}\|_{H^{1}(D)^{2} \otimes L_{2}(\Omega)} & =\|\nabla p\|_{H^{1}(D)^{2} \otimes L_{2}(\Omega)} \\
& \leq\|p\|_{H^{2}(D) \otimes L_{2}(\Omega)} \leq C_{D}\left\|q_{h}-\Theta_{H} q_{h}\right\|_{L_{2}(D) \otimes L_{2}(\Omega)},
\end{aligned}
$$

which, equating $C_{D}$ with $C_{1}$, establishes the lemma.

Theorem 7. For $q_{h} \in Q_{h} \otimes T$,

$$
\left\|q_{h}-\Theta_{H} q_{h}\right\|_{L_{2}(D) \otimes L_{2}(\Omega)} \leq C_{2} H\left\|\operatorname{grad}_{h}^{c} q_{h}\right\|_{U},
$$

where $C_{2}$ is independent of $h, m$, and $n$.

Proof. Define $\nabla \cdot \vec{\sigma}=q_{h}-\Theta_{H} q_{h}$ as in Lemma 1, Then

$$
\begin{aligned}
\left\|q_{h}-\Theta_{H} q_{h}\right\|_{L_{2}(D) \otimes L_{2}(\Omega)}^{2} & =\left(\nabla \cdot \vec{\sigma}, q_{h}-\Theta_{H} q_{h}\right)_{L_{2}(D) \otimes L_{2}(\Omega)} \\
& =\left(\nabla \cdot \vec{\sigma},\left(\Theta_{h}-\Theta_{H}\right) q_{h}\right)_{L_{2}(D) \otimes L_{2}(\Omega)} \\
& =\left(\left(\Theta_{h}-\Theta_{H}\right) \nabla \cdot \vec{\sigma}, q_{h}\right)_{L_{2}(D) \otimes L_{2}(\Omega)} \\
& =\left(\nabla \cdot\left(\Pi_{h}-\Pi_{H}\right) \vec{\sigma}, q_{h}\right)_{L_{2}(D) \otimes L_{2}(\Omega)},
\end{aligned}
$$


where in the last line we used the commutativity result given in (18). Applying the definition of the discrete gradient operator and the Cauchy-Schwarz inequality gives

$$
\begin{aligned}
\left\|q_{h}-\Theta_{H} q_{h}\right\|_{L_{2}(D) \otimes L_{2}(\Omega)}^{2} & =\left(\left(\Pi_{H}-\Pi_{h}\right) \vec{\sigma}, \operatorname{grad}_{h}^{c} q_{h}\right)_{U} \\
& \leq\left\|\left(\Pi_{H}-\Pi_{h}\right) \vec{\sigma}\right\|_{U}\left\|\operatorname{grad}_{h}^{c} q_{h}\right\|_{U} \\
& \leq\left(\left\|\vec{\sigma}-\Pi_{H} \vec{\sigma}\right\|_{U}+\left\|\vec{\sigma}-\Pi_{h} \vec{\sigma}\right\|_{U}\right)\left\|\operatorname{grad}_{h}^{c} q_{h}\right\|_{U} .
\end{aligned}
$$

Now applying the equivalence between $\|\cdot\|_{U}$ and $\|\cdot\|_{L_{2}(D)^{2} \otimes L_{2}(\Omega)}$, the approximation bound given by (13), and noting that $h<H$ gives

$$
\begin{aligned}
\left\|q_{h}-\Theta_{H} q_{h}\right\|_{L_{2}(D) \otimes L_{2}(\Omega)}^{2} \leq & a_{\max }^{1 / 2}\left(\left\|\vec{\sigma}-\Pi_{H} \vec{\sigma}\right\|_{L_{2}(D)^{2} \otimes L_{2}(\Omega)}\right. \\
& \left.+\left\|\vec{\sigma}-\Pi_{h} \vec{\sigma}\right\|_{L_{2}(D)^{2} \otimes L_{2}(\Omega)}\right)\left\|\operatorname{grad}_{h}^{c} q_{h}\right\|_{U} \\
\leq & a_{\max }^{1 / 2}(H+h)\|\vec{\sigma}\|_{H^{1}(D)^{2} \otimes L_{2}(\Omega)}\left\|\operatorname{grad}_{h}^{c} q_{h}\right\|_{U} \\
\leq & a_{\max }^{1 / 2} C_{0} 2 H C_{1}\left\|q_{h}-\Theta_{H} q_{h}\right\|_{L_{2}(D) \otimes L_{2}(\Omega)}\left\|\operatorname{grad}_{h}^{c} q_{h}\right\|_{U},
\end{aligned}
$$

which establishes the theorem with $C_{2}=2 C_{0} C_{1} a_{\max }^{1 / 2}$.

Theorem 8. Let $q_{h} \in Q_{h} \otimes T$ and $\vec{v}_{h}=\operatorname{grad}_{h}^{c} q_{h} \in R_{h} \otimes T$. Define $q_{H}$ and $\vec{v}_{H}$ such that $v_{H}=\operatorname{grad}_{H}^{c} q_{H}$ and $\nabla \cdot \vec{v}_{H}=\Theta_{H} \nabla \cdot \vec{v}_{h}$. Then

$$
\left\|\vec{v}_{h}-\vec{v}_{H}\right\|_{U} \leq C_{3} H\left\|\nabla \cdot \vec{v}_{h}\right\|_{L_{2}(D) \otimes L_{2}(\Omega)},
$$

where $C_{3}$ is independent of $h, m$, and $n$.

Proof. As noted above, $\vec{v}_{h}$ and $\vec{v}_{H}$ can be considered to be finite element approximations to the flux solution of a first-order diffusion problem with source function $f=-\nabla \cdot \operatorname{grad}_{h}^{c} q_{h}$. Let $\vec{v}_{*} \in H(\operatorname{div} ; D) \otimes T$ be the solution to the related semidiscrete problem, as described in 2.9 . Then, using Theorem 1 and noting $H>h$,

$$
\begin{aligned}
\left\|\vec{v}_{h}-\vec{v}_{H}\right\|_{U} & \leq\left\|\vec{v}_{*}-\vec{v}_{h}\right\|_{U}+\left\|\vec{v}_{*}-\vec{v}_{H}\right\|_{U} \leq 2 C_{*} H\left\|\nabla \cdot \operatorname{grad}_{h}^{c} q_{h}\right\|_{L_{2}(D) \otimes L_{2}(\Omega)} \\
& =2 C_{*} H\left\|\nabla \cdot \vec{v}_{h}\right\|_{L_{2}(D) \otimes L_{2}(\Omega)}
\end{aligned}
$$

which establishes the theorem with $C_{3}=2 C_{*}$.

Theorem 9. Let $q_{h} \in Q_{h} \otimes T$ and $\vec{v}_{h}=\operatorname{grad}_{h}^{c} q_{h} \in R_{h} \otimes T$. Define $q_{H}$ and $\vec{v}_{H}$ such that $\vec{v}_{H}=\operatorname{grad}_{H}^{c} q_{H}$ and $\nabla \cdot \vec{v}_{H}=\Theta_{H} \nabla \cdot \vec{v}_{h}$. Then

$$
\left\|\vec{v}_{h}-\vec{v}_{H}\right\|_{\Lambda} \leq C_{4} H\left\|\mathcal{H}_{h} \vec{v}_{h}\right\|_{U}
$$

where $C_{4}$ is independent of $h, m$, and $n$.

Proof. First, note that $\nabla \cdot \vec{v}_{h} \in Q_{h} \otimes T$. Applying Theorem 7 gives

$$
\begin{aligned}
\left\|\nabla \cdot \vec{v}_{h}-\nabla \cdot \vec{v}_{H}\right\|_{L_{2}(D) \otimes L_{2}(\Omega)} & =\left\|\nabla \cdot \vec{v}_{h}-\Theta_{H} \nabla \cdot \vec{v}_{h}\right\|_{L_{2}(D) \otimes L_{2}(\Omega)} \\
& \leq C_{2} H\left\|\operatorname{grad}_{h}^{c} \nabla \cdot \vec{v}_{h}\right\|_{U} .
\end{aligned}
$$

Then, using this along with Theorem 8 gives

$$
\begin{aligned}
\left\|\vec{v}_{h}-\vec{v}_{H}\right\|_{\Lambda}^{2} & =\left\|\vec{v}_{h}-\vec{v}_{H}\right\|_{U}^{2}+\left\|\nabla \cdot \vec{v}_{h}-\nabla \cdot \vec{v}_{H}\right\|_{L_{2}(D) \otimes L_{2}(\Omega)}^{2} \\
& \leq C_{3}^{2} H^{2}\left\|\nabla \cdot \vec{v}_{h}\right\|_{L_{2}(D) \otimes L_{2}(\Omega)}^{2}+C_{2}^{2} H^{2}\left\|\operatorname{grad}_{h}^{c} \nabla \cdot \vec{v}_{h}\right\|_{U}^{2} .
\end{aligned}
$$

It can be shown, using the definition of the weighted $H(\operatorname{div} ; D) \otimes L_{2}(\Omega)$ operator given in 3.3 , that

$$
\left\|\mathcal{H}_{h} \vec{v}_{h}\right\|_{U}^{2}=\left\|\vec{v}_{h}\right\|_{\Lambda}^{2}+\left\|\nabla \cdot \vec{v}_{h}\right\|_{L_{2}(D) \otimes L_{2}(\Omega)}^{2}+\left\|\operatorname{grad}_{h}^{c} \nabla \cdot \vec{v}_{h}\right\|_{U}^{2} .
$$


Therefore,

$$
\left\|\vec{v}_{h}-\vec{v}_{H}\right\|_{\Lambda} \leq \max \left\{C_{2}, C_{3}\right\} H\left\|\mathcal{H}_{h} \vec{v}_{h}\right\|_{U},
$$

which establishes the theorem with $C_{4}=\max \left\{C_{2}, C_{3}\right\}$.

5.2. $\Lambda$-projection bounds. In this section we obtain two bounds involving a function in $R_{h} \otimes T$ and its projection with respect to $\Lambda(\cdot, \cdot)$ onto $R_{H} \otimes T$. These two bounds comprise the analogue of Lemma 3.2 in [1].

Theorem 10. Let $\vec{v} \in R_{h} \otimes T$ and define $\vec{w}=\vec{v}-\mathcal{P}_{H} \vec{v} \in R_{h} \otimes T$ with Helmholtz Decomposition $\vec{w}=\operatorname{grad}_{h}^{c} q+\nabla \times z$. Then

$$
\left\|\operatorname{grad}_{h}^{c} q\right\|_{U} \leq C_{4} H\|\vec{w}\|_{\Lambda},
$$

where $C_{4}$ is as in Theorem 9 ,

Proof. First note that

$$
\Lambda\left(\operatorname{grad}_{h}^{c} q, \mathcal{H}_{h}^{-1} \operatorname{grad}_{h}^{c} q\right)=\left(\operatorname{grad}_{h}^{c} q, \operatorname{grad}_{h}^{c} q\right)_{U}=\left\|\operatorname{grad}_{h}^{c} q\right\|_{U}^{2} .
$$

Let $\vec{\tau}_{h}=\mathcal{H}_{h}^{-1} \operatorname{grad}_{h}^{c} q$. Now, using (15), we have

$$
\begin{aligned}
\Lambda\left(\nabla \times z, \vec{\tau}_{h}\right) & =\int_{\Omega} \int_{D} \frac{1}{c} \nabla \times z \cdot \vec{\tau}_{h}+\int_{\Omega} \int_{D}(\nabla \cdot \nabla \times z)\left(\nabla \cdot \vec{\tau}_{h}\right) \\
& =\int_{\Omega} \int_{D} \frac{1}{c} \nabla \times z \cdot \vec{\tau}_{h}=\left(\nabla \times z, \vec{\tau}_{h}\right)_{U} .
\end{aligned}
$$

As $\mathcal{H}_{h}$ (and hence $\mathcal{H}_{h}^{-1}$ ) maps $\operatorname{grad}_{h}^{c}\left(Q_{h} \otimes T\right.$ ) onto itself (as noted in 33.3 ) there exists $q^{\prime} \in Q_{h} \otimes T$ such that $\vec{\tau}_{h}=\operatorname{grad}_{h}^{c} q^{\prime}$. So, using (16),

$$
\Lambda\left(\nabla \times z, \vec{\tau}_{h}\right)=\left(\nabla \times z, \operatorname{grad}_{h}^{c} q^{\prime}\right)_{U}=-\left(\nabla \cdot \nabla \times z, q^{\prime}\right)_{L_{2}(D) \otimes L_{2}(\Omega)}=0,
$$

on account of (15) again. Define $\vec{\tau}_{H}$ in relation to $\vec{\tau}_{h}$ as in Theorem 9, Then

$$
\begin{aligned}
\Lambda\left(\operatorname{grad}_{h}^{c} q, \vec{\tau}_{h}\right) & =\Lambda\left(\vec{v}-\mathcal{P}_{H} \vec{v}, \vec{\tau}_{h}\right)=\Lambda\left(\vec{v}-\mathcal{P}_{H} \vec{v}, \vec{\tau}_{h}-\vec{\tau}_{H}\right) \\
& \leq\left\|\vec{v}-P_{H} \vec{v}\right\|_{\Lambda}\left\|\vec{\tau}_{h}-\vec{\tau}_{H}\right\|_{\Lambda} \leq C_{4} H\|\vec{w}\|_{\Lambda}\left\|\operatorname{grad}_{h}^{c} q\right\|_{U},
\end{aligned}
$$

which completes the proof.

Theorem 11. Let $\vec{v} \in R_{h} \otimes T$ and define $\vec{w}=\vec{v}-\mathcal{P}_{H} \vec{v} \in R_{h} \otimes T$ with Helmholtz Decomposition $\vec{w}=\operatorname{grad}_{h}^{c} q+\nabla \times z$. Then

$$
\|z\|_{L_{2}(D) \otimes L_{2}(\Omega)} \leq C_{5} H\|\vec{w}\|_{\Lambda},
$$

where $C_{5}$ is independent of $h, m$, and $n$.

Proof. A standard result from the deterministic analysis (cf. [1) yields

$$
\|z\|_{L_{2}(D)} \leq C_{6} H\|\nabla \times z\|_{L_{2}(D)^{2}} \quad \forall \omega \in \Omega
$$

for some constant $C_{6}$. Therefore,

$$
\left\|\left.z\right|_{L_{2}(D) \otimes L_{2}(\Omega)} \leq C_{6} H\right\| \nabla \times z\left\|_{L_{2}(D)^{2} \otimes L_{2}(\Omega)} \leq C_{6} a_{\min }^{-1 / 2} H\right\| \nabla \times z \|_{U} .
$$

Now, using the properties of the Helmholtz decomposition noted in 3.1 .

$$
\begin{aligned}
\|\nabla \times z\|_{U}^{2} & =\left(\vec{w}-\operatorname{grad}_{h}^{c} q, \nabla \times z\right)_{U}=(\vec{w}, \nabla \times z)_{U} \\
& =(\vec{w}, \nabla \times z)_{U}+(\nabla \cdot \vec{w}, \nabla \cdot \nabla \times z)_{L_{2}(D) \otimes L_{2}(D)} \\
& =\Lambda(\vec{w}, \nabla \times z) \leq\|\vec{w}\|_{\Lambda}\|\nabla \times z\|_{U} .
\end{aligned}
$$

Therefore $\|\nabla \times z\|_{U} \leq\|\vec{w}\|_{\Lambda}$, which combined with the above result establishes the theorem with $C_{5}=C_{6} a_{\min }^{-1 / 2}$. 


\section{Additive Schwarz method}

A constitutive part of multigrid is the smoother, where by smoother we mean an iterative method that successively smoothes the error associated with the iterates of the system that multigrid is applied to. Following [1] we will use the additive Schwarz method (extended to incorporate the random nature of the problem) as our smoother. This method is defined with respect to a decomposition of the spatial domain $D$. In this section we will define the additive Schwarz operator and establish some results that will ultimately be used to demonstrate the convergence of the multigrid algorithm defined in 97.1 .

6.1. Domain decomposition results. Let $\mathcal{D}=\left\{D_{1}, \ldots, D_{L}\right\}$ be an overlapping covering of $D$. Let $\gamma$ be an integer such that no point in $\bar{D}$ occurs in more than $\gamma$ elements of $\mathcal{D}$. Then $\sum_{k} \int_{D_{k}} \leq \gamma \int_{D}$ and $\gamma$ is called the overlap parameter. Though we need not be specific here we note that in practice we will take each element of $\mathcal{D}$ to be a set of triangles in $\mathcal{T}$ that share a common node. For the partitions that we use to generate the numerical results in $₫ 8$ this gives $\gamma=3$. Now define

$$
R^{k} \otimes T=\left\{\vec{v} \in R \otimes T \mid \operatorname{supp}(v) \subset D_{k} \times \Omega\right\} .
$$

Given $\vec{v} \in R \otimes T$ there exists a decomposition $\vec{v}=\sum_{k} \vec{v}_{k}$ where $\vec{v}_{k} \in R^{k} \otimes T$. Let $\left\{\theta^{k}: \bar{D} \rightarrow \mathbb{R}\right\}_{k=1}^{L}$ be a partition of unity subordinate to the covering $\mathcal{D}$, i.e. $\sum_{k} \theta^{k}=1$ and $\operatorname{supp}\left(\theta^{k}\right) \subset D_{k}$.

Theorem 12. Let $\vec{v} \in R \otimes T$ and $\vec{v}_{k}=\Pi \theta^{k} \vec{v} \in R^{k} \otimes T$. Then $\vec{v}=\sum_{k} \vec{v}_{k}$ and

$$
\sum_{k=1}^{L} \Lambda\left(\vec{v}_{k}, \vec{v}_{k}\right) \leq C_{7}\left(\left(1+h^{-2}\right)\|\vec{v}\|_{U}^{2}+\|\nabla \cdot \vec{v}\|_{L_{2}(D) \otimes L_{2}(\Omega)}^{2}\right),
$$

where $C_{7}$ is a constant.

Proof. Following [1, there exists a constant $C_{8}$ such that

$$
\left\|\vec{v}_{k}\right\|_{L_{2}(D)^{2}} \leq C_{8}\|\vec{v}\|_{L_{2}\left(D_{k}\right)^{2}} \quad \forall \omega \in \Omega .
$$

Introducing the notation $\|\cdot\|_{U_{k}}$ to denote a norm defined in the same manner as $\|\cdot\|_{U}$ but restricted to $D_{k}$, we have

$$
\left\|\vec{v}_{k}\right\|_{U}^{2} \leq a_{\max }\left\|\vec{v}_{k}\right\|_{L_{2}(D)^{2} \otimes L_{2}(\Omega)}^{2} \leq C_{8}^{2} a_{\max }\|\vec{v}\|_{L_{2}\left(D_{k}\right)^{2} \otimes L_{2}(\Omega)}^{2} \leq \frac{C_{8}^{2} a_{\max }}{a_{\min }}\|\vec{v}\|_{U_{k}}^{2} .
$$

Also following [1] there exists a constant $C_{9}$ such that

$$
\left\|\nabla \cdot \vec{v}_{k}\right\|_{L_{2}(D)} \leq C_{9}\left(h^{-1}\|\vec{v}\|_{L_{2}\left(D_{k}\right)^{2}}+\|\nabla \cdot \vec{v}\|_{L_{2}\left(D_{k}\right)}\right) \quad \forall \omega \in \Omega .
$$

Therefore,

$$
\left\|\nabla \cdot \vec{v}_{k}\right\|_{L_{2}(D) \otimes L_{2}(\Omega)}^{2} \leq 2 C_{9}^{2}\left(h^{-2} a_{\min }^{-1}\|\vec{v}\|_{U_{k}}^{2}+\|\nabla \cdot \vec{v}\|_{L_{2}(D) \otimes L_{2}(\Omega)}^{2}\right) .
$$

Then, using these two results, we have

$$
\begin{aligned}
& \sum_{k=1}^{L} \Lambda\left(\vec{v}_{k}, \vec{v}_{k}\right)=\sum_{k=1}^{L}\left(\left\|\vec{v}_{k}\right\|_{U}^{2}+\left\|\nabla \cdot \vec{v}_{k}\right\|_{L_{2}(D) \otimes L_{2}(\Omega)}^{2}\right) \\
& \leq \sum_{k=1}^{L}\left(\frac{C_{8}^{2} a_{\max }}{a_{\min }}\left\|\vec{v}_{k}\right\|_{U_{k}}^{2}+2 C_{9}^{2}\left(\frac{1}{h^{2} a_{\min }}\|\vec{v}\|_{U_{k}}^{2}+\|\nabla \cdot \vec{v}\|_{L_{2}\left(D_{k}\right) \otimes L_{2}(\Omega)}^{2}\right)\right),
\end{aligned}
$$


which establishes the theorem with

$$
C_{7}=\gamma \max \left\{\frac{C_{8}^{2} a_{\max }}{a_{\min }}, 2 C_{9} \max \left\{\frac{1}{a_{\min }}, 1\right\}\right\},
$$

where $\gamma$ is the overlap parameter noted above.

6.2. Additive Schwarz operator. We define the projection operator $\mathcal{P}^{k}: R \otimes$ $T \rightarrow R^{k} \otimes T$ such that for $\vec{v} \in R \otimes T$,

$$
\Lambda\left(\mathcal{P}^{k} \vec{v}, \vec{w}\right)=\Lambda(\vec{v}, \vec{w}) \quad \forall \vec{w} \in R^{k} \otimes T .
$$

Note the similarity between $\mathcal{P}^{k}$ and the projection operator $\mathcal{P}$ defined in $\$ 3.2$. The additive Schwarz operator is now defined by

$$
\mathcal{S}=\eta \sum_{k=1}^{L} \mathcal{P}^{k} \mathcal{H}^{-1},
$$

where $\eta$ is some constant chosen such that Theorem 13 below holds.

The additive Schwarz operator thus defined is symmetric with respect to $(\cdot, \cdot)_{U}$. To see this let $\vec{v}, \vec{w} \in R \otimes T$. Then

$$
\begin{aligned}
(\mathcal{S} \vec{v}, \vec{w})_{U} & =\eta \sum_{k=1}^{L}\left(\mathcal{P}^{k} \mathcal{H}^{-1} \vec{v}, \vec{w}\right)_{U}=\eta \sum_{k=1}^{L} \Lambda\left(\mathcal{P}^{k} \mathcal{H}^{-1} \vec{v}, \mathcal{H}^{-1} \vec{w}\right) \\
& =\eta \sum_{k=1}^{L} \Lambda\left(\mathcal{H}^{-1} \vec{v}, \mathcal{P}^{k} \mathcal{H}^{-1} \vec{w}\right)=\Lambda\left(\mathcal{H}^{-1} \vec{v}, \mathcal{S} \vec{w}\right)=(\vec{v}, \mathcal{S} \vec{w})_{U}
\end{aligned}
$$

The operator $\mathcal{S}$ can also be shown to be positive definite with respect to $(\cdot, \cdot)_{U}$ provided that, given $\vec{v} \in R \otimes T$, there exists a decomposition $\vec{v}=\sum_{k=1}^{L} \vec{v}_{k}, \vec{v}_{k} \in$ $R^{k} \otimes T$ such that

$$
\sum_{k=1}^{L} \Lambda\left(\vec{v}_{k}, \vec{v}_{k}\right) \leq C_{\diamond} \Lambda(\vec{v}, \vec{v}),
$$

where $C_{\diamond}$ is a positive constant. To see this, we employ a standard argument as given, e.g., in 21]. Let $\mathcal{P}_{\mathcal{S}}=\sum_{k=1}^{L} \mathcal{P}^{k}$. Then

$$
\begin{aligned}
\Lambda(\vec{v}, \vec{v}) & =\sum_{k=1}^{L} \Lambda\left(\vec{v}, \vec{v}_{k}\right)=\sum_{k=1}^{L} \Lambda\left(\mathcal{P}^{k} \vec{v}, \vec{v}_{k}\right) \leq \sum_{k=1}^{L}\left\|\mathcal{P}^{k} \vec{v}\right\|_{\Lambda}\left\|\vec{v}_{k}\right\|_{\Lambda} \\
& \leq\left(\sum_{k=1}^{L} \Lambda\left(\mathcal{P}^{k} \vec{v}, \mathcal{P}^{k} \vec{v}\right)\right)^{1 / 2}\left(\sum_{k=1}^{L} \Lambda\left(\vec{v}_{k}, \vec{v}_{k}\right)\right)^{1 / 2} \\
& \leq\left(\Lambda\left(\mathcal{P}_{\mathcal{S}} \vec{v}, \vec{v}\right)\right)^{1 / 2}\left(C_{\diamond} \Lambda(\vec{v}, \vec{v})\right)^{1 / 2} .
\end{aligned}
$$

Therefore,

$$
\Lambda\left(\mathcal{P}_{\mathcal{S}} \vec{v}, \vec{v}\right) \geq \frac{1}{C_{\diamond}} \Lambda(\vec{v}, \vec{v})
$$


Returning to the additive Schwarz operator and letting $\vec{w}=\mathcal{H}^{-1} \vec{v}$ gives

$$
\begin{aligned}
(\mathcal{S} \vec{v}, \vec{v})_{U} & =\eta \sum_{k=1}^{L}\left(\mathcal{P}^{k} \mathcal{H}^{-1} \vec{v}, \vec{v}\right)_{U}=\eta \sum_{k=1}^{L} \Lambda\left(\mathcal{P}^{k} \mathcal{H}^{-1} \vec{v}, \mathcal{H}^{-1} \vec{v}\right) \\
& =\eta \sum_{k=1}^{L} \Lambda\left(\mathcal{P}^{k} \vec{w}, \vec{w}\right)=\eta \Lambda\left(\mathcal{P}_{\mathcal{S}} \vec{w}, \vec{w}\right) \geq \frac{1}{C_{\diamond}} \eta \Lambda(\vec{w}, \vec{w})
\end{aligned}
$$

As $\vec{v}=0 \Leftrightarrow \vec{w}=0$ this shows that $\mathcal{S}$ is positive definite and hence invertible. That (26) holds for all $v \in R \otimes T$ can be shown by following a similar line of reasoning as in [1]. However, here we show that (26) holds on a necessary subspace of $R \otimes T$. This is done in the following section.

\section{Multigrid}

In the following we consider a family of triangulations $\mathcal{T}_{j}, j=1, \ldots, J$, with mesh parameters $h_{j}$, where $h_{i}>h_{j}$ for $i<j$. We assume these triangulations give rise to a nested sequence of finite-dimensional spaces denoted by $R_{1} \otimes T \subset \cdots \subset R_{J} \otimes T \subset$ $U$, as defined in $\$ 2.6$. For the remainder of this section the various operators and spaces defined in $\S ₫ 2$, 3 will carry a subscript to denote the underlying triangulation on which they are defined. We let $\mathcal{D}_{j}$ be a covering of $D$ defined with respect to $\mathcal{T}_{j}$, as discussed in 6.1 , and we let $\mathcal{S}_{j}, j=2, \ldots, J$, be the additive Schwarz operator defined with respect to $\mathcal{D}_{j}$.

7.1. Multigrid V-cycle. We now define a multigrid algorithm, analogous to that given in [1, for solving the vector equation $\mathcal{H}_{J} \vec{v}=\vec{z}$. Let $\vec{z} \in R_{k} \otimes T$. Then we define $\mathcal{V}_{k}^{-1}: R_{k} \otimes T \rightarrow R_{k} \otimes T$ such that $\mathcal{V}_{1}^{-1} \vec{z}=\mathcal{H}_{1}^{-1} \vec{z}$ and $\mathcal{V}_{k}^{-1} \vec{z}, k=2, \ldots, J$, by the recursive algorithm:

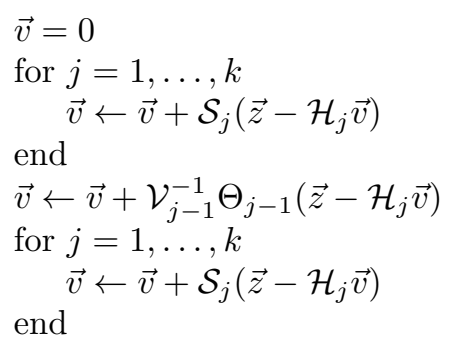

This corresponds to a multigrid V-cycle with one multigrid iteration at each level. Moreover, at each level there are $k$ pre-smoothing and post-smoothing steps.

The matrix representation of the operator $\mathcal{H}_{J}$ is $A_{J}^{-1}\left(A_{J}+F_{J}\right)$, where $A_{J}$ and $F_{J}$ are as in 4.2 Therefore, the vector equation $\mathcal{H}_{J} \vec{v}=\vec{z}$ can be expressed as the linear system $\left(A_{J}+F_{J}\right) \mathbf{v}=A_{J} \mathbf{z}$, where $\mathbf{v}$ and $\mathbf{z}$ are the coefficient vectors of $\vec{v}$ and $\vec{z}$. This relationship along with the convergence properties of the above algorithm will be used in $\$ 7.2$ to demonstrate that the spectral bounds in (24) will be independent of the parameters $h, m$, and $n$ if the matrix $V_{J}$ is chosen such that $V_{J}^{-1}$ is the matrix representation of the multigrid operator $\mathcal{V}_{J}^{-1}$. 
7.2. Multigrid convergence. We here establish that the multigrid algorithm given in $\$ 7.1$ converges and that $\mathcal{V}_{J}$ and $\mathcal{H}_{J}$ are spectrally equivalent.

Theorem 13. The inequality

$$
\Lambda\left(\left(I-\mathcal{S}_{j} \mathcal{H}_{j}\right) \vec{v}, \vec{v}\right) \geq 0 \quad \forall \vec{v} \in R_{j} \otimes T
$$

holds for $j=1, \ldots, J$.

Proof. For $j=1, \mathcal{S}_{j}=\mathcal{H}_{j}^{-1}$ and the result holds as $\Lambda(\cdot, \cdot)$ is an inner product on $R_{1} \otimes T$. Let $2<j \leq J$. Then

$$
\begin{aligned}
\Lambda\left(\left(I-\mathcal{S}_{j} \mathcal{H}_{j}\right) \vec{v}, \vec{v}\right) & =\Lambda(\vec{v}, \vec{v})-\Lambda\left(\mathcal{S}_{j} \mathcal{H}_{j} \vec{v}, \vec{v}\right)=\Lambda(\vec{v}, \vec{v})-\eta \sum_{k=1}^{L_{j}} \Lambda\left(\mathcal{P}_{j}^{k} \vec{v}, \vec{v}\right) \\
& =\Lambda(\vec{v}, \vec{v})-\eta \sum_{k=1}^{L_{j}} \Lambda\left(\mathcal{P}_{j}^{k} \vec{v}, \mathcal{P}_{j}^{k} \vec{v}\right)=\Lambda(\vec{v}, \vec{v})-\eta \sum_{k=1}^{L_{j}}\left\|\mathcal{P}_{j}^{k} \vec{v}\right\|_{\Lambda}^{2} .
\end{aligned}
$$

Let $\|\cdot\|_{\Lambda_{k}}$ be the norm induced on $D_{k} \times \Omega$ by $\|\cdot\|_{\Lambda}$. Then

$$
\left\|\mathcal{P}_{j}^{k} \vec{v}\right\|_{\Lambda}^{2}=\Lambda\left(\mathcal{P}_{j}^{k} \vec{v}, \mathcal{P}_{j}^{k} \vec{v}\right)=\Lambda\left(\mathcal{P}_{j}^{k} \vec{v}, \vec{v}\right) \leq\left\|\mathcal{P}_{j}^{k} \vec{v}\right\|_{\Lambda_{k}}\|\vec{v}\|_{\Lambda_{k}}=\left\|\mathcal{P}_{j}^{k} \vec{v}\right\|_{\Lambda}\|\vec{v}\|_{\Lambda_{k}}
$$

Therefore,

$$
\Lambda\left(I-\mathcal{S}_{j} \mathcal{H}_{j} \vec{v}, \vec{v}\right) \leq(1-\eta \gamma) \Lambda(\vec{v}, \vec{v})
$$

where $\gamma$ is the overlap parameter discussed in $\$ 6.1$. So the theorem holds providing $\eta \leq \gamma^{-1}$.

Lemma 2. Let $\vec{v} \in R_{j} \otimes T$ and let $\vec{w} \in\left(I-\mathcal{P}_{j-1}\right) \vec{v} \in R_{j} \otimes T$. Then, for $j=2, \ldots, J$, there exists a decomposition $\vec{w}=\sum_{k=1}^{L_{j}} \vec{w}_{k}, \vec{w}_{k} \in R_{j}^{k} \otimes T$ such that

$$
\sum_{k=1}^{L_{j}} \Lambda\left(\vec{w}_{k}, \vec{w}_{k}\right) \leq C_{10} \Lambda(\vec{w}, \vec{w})
$$

where $C_{10}$ is independent of $h, m$, and $n$.

Proof. Let $\vec{w}$ have the Helmholtz decomposition $\vec{w}=\operatorname{grad}_{h_{j}}^{c} q+\nabla \times s$, where $q \in Q_{j} \otimes T$ and $s \in W_{j} \otimes T$. Define $\vec{w}^{\prime}=\operatorname{grad}_{h_{j}}^{c} q$ and $\vec{w}^{\prime \prime}=\nabla \times s$. Then given the decompositions $\vec{w}^{\prime}=\sum_{k} \vec{w}_{k}^{\prime}$ and $\vec{w}^{\prime \prime}=\sum_{k} \vec{w}_{k}^{\prime \prime}$ and taking into account that the Helmholtz decomposition is orthogonal with respect to $\Lambda(\cdot, \cdot)$, it is sufficient to show that

$$
\sum_{k=1}^{L_{j}} \Lambda\left(\vec{w}_{k}^{\prime}, \vec{w}_{k}^{\prime}\right) \leq C_{10}^{\prime} \Lambda(\vec{w}, \vec{w}), \quad \sum_{k=1}^{L_{j}} \Lambda\left(\vec{w}_{k}^{\prime \prime}, \vec{w}_{k}^{\prime \prime}\right) \leq C_{10}^{\prime \prime} \Lambda(\vec{w}, \vec{w})
$$

for some constants $C_{10}^{\prime}$ and $C_{10}^{\prime \prime}$. This will result in $C_{10}=2 \max \left\{C_{10}^{\prime}, C_{10}^{\prime \prime}\right\}$. To show the first of these, let $w_{k}^{\prime}=\Pi_{j} \theta_{j}^{k} w^{\prime}$, where $\theta_{j}^{k}$ is as in $\$ 6.2$ Invoking Theorems 10 and 12 gives

$$
\begin{aligned}
\sum_{k=1}^{L_{j}} \Lambda\left(\vec{w}_{k}^{\prime}, \vec{w}_{k}^{\prime}\right) & \leq C_{7}\left(\left(1+h_{j}^{-2}\right)\left\|\vec{w}^{\prime}\right\|_{U}^{2}+\left\|\nabla \cdot \vec{w}^{\prime}\right\|_{L_{2}(D) \otimes L_{2}(\Omega)}^{2}\right) \\
& =C_{7}\left(\left\|\vec{w}^{\prime}\right\|_{\Lambda}^{2}+h_{j}^{-2}\left\|\vec{w}^{\prime}\right\|_{U}^{2}\right) \leq C_{7}\left(\left\|\vec{w}^{\prime}\right\|_{\Lambda}^{2}+C_{4}^{2} h_{j}^{-2} h_{j-1}^{2}\|\vec{w}\|_{\Lambda}^{2}\right) .
\end{aligned}
$$


Assume that for $j=2, \ldots, J$, there exists $\varrho>h_{j-1} / h_{j}$. Then, as $\left\|\vec{w}^{\prime}\right\|_{\Lambda} \leq\|\vec{w}\|_{\Lambda}$,

$$
\sum_{k=1}^{L_{j}} \Lambda\left(\vec{w}_{k}^{\prime}, \vec{w}_{k}^{\prime}\right) \leq 2 C_{7} \max \left\{1, C_{4}^{2} \varrho^{2}\right\} \Lambda(\vec{w}, \vec{w}) .
$$

Next, following [1], there exists a decomposition $\vec{w}^{\prime \prime}=\sum \vec{w}_{k}^{\prime \prime}$ such that

$$
\sum_{k=1}^{L_{j}}\left(\vec{w}_{k}^{\prime \prime}, \vec{w}_{k}^{\prime \prime}\right)_{L_{2}(D)^{2}} \leq C_{11}\left(\left\|\vec{w}^{\prime \prime}\right\|_{L_{2}(D)^{2}}^{2}+h_{j}^{-2}\|s\|_{L_{2}(D)}^{2}\right) \quad \forall \omega \in \Omega,
$$

where $C_{11}$ is a constant. From this it follows that

$$
\sum_{k=1}^{L_{j}} \Lambda\left(\vec{w}_{k}^{\prime \prime}, \vec{w}_{k}^{\prime \prime}\right) \leq a_{\max } C_{11}\left(a_{\min }^{-1}\left\|w^{\prime \prime}\right\|_{\Lambda}^{2}+h_{j}^{-2}\|s\|_{L_{2}(D) \otimes L_{2}(\Omega)}^{2}\right) .
$$

Applying Theorem 11 and noting that $\left\|\vec{w}^{\prime \prime}\right\|_{\Lambda} \leq\|\vec{w}\|_{\Lambda}$ gives

$$
\sum_{k=1}^{L_{j}} \Lambda\left(\vec{w}_{k}^{\prime \prime}, \vec{w}_{k}^{\prime \prime}\right) \leq 2 a_{\max } C_{11} \max \left\{a_{\min }^{-1}, C_{5}^{2} \varrho^{2}\right\} \Lambda(\vec{w}, \vec{w}) .
$$

Therefore the theorem is established.

Lemma 3. Let $\vec{v} \in R_{j} \otimes T$ and $\vec{w}=\left(I-\mathcal{P}_{j-1}\right) \vec{v}$. Then for $j=2, \ldots, J$,

$$
\left(\mathcal{S}_{j}^{-1} \vec{w}, \vec{w}\right)_{U}=\frac{1}{\eta} \inf \sum_{k=1}^{L_{j}} \Lambda\left(\vec{w}_{k}, \vec{w}_{k}\right),
$$

where the infinum is taken over all decompositions of the form $\vec{w}=\sum_{k} \vec{w}_{k}, \vec{w}_{k} \in$ $R_{j}^{k} \otimes T$.

Proof. Let $E_{j}=\left\{\vec{w} \in R_{j} \otimes T \mid \vec{w}=\left(I-\mathcal{P}_{j-1}\right) \vec{v}, \vec{v} \in R_{j} \otimes T\right\}$ for $j=2, \ldots, J$. As a consequence of Lemma 2, (24) is satisfied on each of these sets and, therefore, $\mathcal{S}_{j}$ is invertible on $E_{j}$. The rest of the proof follows in an analogous fashion to the proof for the deterministic case given in Appendix B of [1].

Theorem 14. The inequality

$\left(\mathcal{S}_{j}^{-1}\left(I-\mathcal{P}_{j-1}\right) \vec{v},\left(I-\mathcal{P}_{j-1}\right) \vec{v}\right)_{U} \leq C_{12} \Lambda\left(\left(I-\mathcal{P}_{j-1}\right) \vec{v},\left(I-\mathcal{P}_{j-1}\right) \vec{v}\right) \quad \forall \vec{v} \in R_{j} \otimes T$ holds for $j=2, \ldots, J$, where $C_{12}$ is independent of $h, m$, and $n$.

Proof. Let $\vec{w}=\left(I-\mathcal{P}_{j-1}\right) \vec{v}$. Then for each $\vec{v} \in R_{j} \otimes T$ it is required to show that

$$
\left(\mathcal{S}^{-1} \vec{w}, \vec{w}\right)_{U} \leq C_{12} \Lambda(\vec{w}, \vec{w}) .
$$

From Lemma 2 we know that there exists a decomposition $\vec{w}=\sum_{k} \vec{w}_{k}, \vec{w}_{k} \in R_{j}^{k} \otimes T$ such that

$$
\sum_{k=1}^{L_{j}} \Lambda\left(\vec{w}_{k}, \vec{w}_{k}\right) \leq C_{10} \Lambda(\vec{w}, \vec{w}) .
$$

Now using Lemma 3 gives the required inequality with $C_{12}=\eta^{-1} C_{10}$.

Theorem 15. The eigenvalues of $\mathcal{V}_{J}^{-1} \mathcal{H}_{J}$ are contained in the interval $[1-\delta, 1]$, where

$$
\delta=\frac{C_{12}}{C_{12}+2 k}
$$


Proof. Given Theorem 13 and Theorem [15, the proof follows that for Corollary 5.2 in [1].

Note that $\delta$, thus defined, depends on $k$, which denotes the number of presmoothing and post-smoothing steps in the multigrid algorithm, and $C_{12}=\eta^{-1} C_{10}$, where $C_{10}$ is independent of the discretization parameters, $h, m$, and $n$, but does depend on the lower and upper bounds of the diffusion coefficient, $a_{\min }$ and $a_{\max }$.

From Theorem 15 we have

$$
1-\delta \leq \frac{\left(\mathcal{H}_{J} \vec{v}, \vec{v}\right)_{U}}{\left(\mathcal{V}_{J} \vec{v}, \vec{v}\right)_{U}} \leq 1 \quad \forall \vec{v} \in V_{J}
$$

As noted in $\$ 7.1$ the multigrid algorithm finds an approximation, $\vec{v}_{1}$ say, to the solution of $\mathcal{H}_{J} \vec{v}=\vec{z}$. Moreover, $\mathcal{H}_{J} \vec{v}=\vec{z}$ can be expressed as $\left(A_{J}+F_{J}\right) \mathbf{v}=A_{J} \mathbf{z}$, where $A_{J}$ and $F_{J}$ are as in 44.2 and $\mathbf{v}$ and $\mathbf{z}$ are the coefficient vectors of $\vec{v}$ and $\vec{z}$. Now we define the matrix $V_{J}$ such that $V_{J}^{-1} A_{J} \mathbf{z}=\mathbf{v}_{1}$, where $\mathbf{v}_{1}$ is the coefficient vector of $\vec{v}_{1}$. That is to say, $V_{J}^{-1}$ is the matrix representation of the multigrid operator $\mathcal{V}_{J}^{-1}$. With $V_{J}$ defined in this way we have

$$
\frac{\left(\mathcal{H}_{J} \vec{v}, \vec{v}\right)_{U}}{\left(\mathcal{V}_{J} \vec{v}, \vec{v}\right)_{U}}=\frac{\Lambda(\vec{v}, \vec{v})}{\left(\mathcal{V}_{J} \vec{v}, \vec{v}\right)_{U}}=\frac{\left(\left(A_{J}+F_{J}\right) \mathbf{v}, \mathbf{v}\right)}{\left(A_{J}^{-1} V_{J} \mathbf{v}, \mathbf{v}\right)_{A_{J}}}=\frac{\left(\left(A_{J}+F_{J}\right) \mathbf{v}, \mathbf{v}\right)}{\left(V_{J} \mathbf{v}, \mathbf{v}\right)}
$$

Therefore, (24) is satisfied with $\theta=1-\delta$ and $\bar{\theta}=1$. Consequently, as $\theta$ governs the values of the eigenvalue bounds described in Theorem [6, we expect the preconditioned MINRES scheme given in 4.2 to converge independently of the discretization parameters but not of the upper and lower bounds of the diffusion coefficient.

\section{NUMERICAL IMPLEMENTATION}

Our aim is to solve (12) via MINRES using the preconditioner (25). Recall that $N$ is a diagonal matrix and the action of $V^{-1}$ is achieved by applying a $\mathrm{V}$-cycle of the multigrid algorithm analyzed in $\S 7$ to a system with the coefficient matrix

$$
A+F=G_{0} \otimes\left(K_{0}+D_{0}\right)+\sum_{r=1}^{m} \sqrt{\lambda_{r}} G_{r} \otimes K_{r} .
$$

The cost of computing the action of $V^{-1}$ is clearly a crucial consideration, and we now offer some practical insight into this.

The intergrid transfer operators are defined in the usual way. That is, prolongation is achieved via natural inclusion and restriction is defined via transposition. To describe the additive Schwarz smoother, let $H_{j}=A_{j}+F_{j}$ denote the stochastic weighted $H$ (div) matrix associated with the triangulation $\mathcal{T}_{j}$. This triangulation is decomposed into $L_{j}$ overlapping patches which constitute the elements of $\mathcal{D}_{j}$ as described in $\$ 6.1$. Then the matrix representation of $\mathcal{S}_{j}$ is given by

$$
S_{j}=\eta \sum_{k=1}^{L_{j}} P_{j}^{k} H_{j}^{-1}, \quad P_{j}^{k}=\left(I \otimes J_{j}^{k}\right)^{T}\left(H_{j}^{k}\right)^{-1}\left(I \otimes J_{j}^{k}\right) H_{j},
$$

where $\left(I \otimes J_{j}^{k}\right)$ is the patch restriction matrix, $I$ is the $M \times M$ identity matrix, and $J_{j}^{k} \in \mathbb{R}^{N_{R_{j}} \times N_{R_{j}}}$ is the standard matrix with entries zero or one that extracts the components of $\mathbf{w} \in \mathbb{R}^{N_{R_{j}}}$ which are associated with the $k$-th patch in $\mathcal{D}_{j}$. Following the advice in [1, we choose the scaling parameter in the definition of $S_{j}$ to be $\eta=1 / 2$. 
Now, computing $S_{j} \mathbf{v}$, where $\mathbf{v} \in \mathbb{R}^{M N_{R_{j}}}$, requires local solves with the matrices

$$
H_{j}^{k}=G_{0} \otimes\left(K_{0, j}^{k}+D_{0, j}^{k}\right)+\sum_{r=1}^{m} \sqrt{\lambda_{r}} G_{r} \otimes K_{r, j}^{k}, \quad k=1, \ldots, L_{j},
$$

where $K_{0, j}^{k}, D_{0, j}^{k}$, and $K_{r, j}^{k}$ are small principal submatrices of $K_{0, j}, D_{0, j}$, and $K_{r, j}$, respectively. The major strength of this method is that the $L_{j}$ solves are decoupled and can (and should) be performed in parallel. The size of $H_{j}^{k}$ is approximately $6 M \times 6 M$, where $M$ is defined in (8) and six is the typical number of flux degrees of freedom lying on a patch in $\mathcal{D}_{j}$. We assume that these $L_{j}$ systems are solved via direct methods and so a few thousand stochastic degrees of freedom can be handled comfortably in a parallel computing environment.

If parallel computing facilities are not available or $M$ is in the order of hundreds of thousands or more, then a cheaper smoother may have to be considered. We outline one possibility. Suppose that we define a modified smoother $\hat{S}_{j}$ via

$$
\hat{S}_{j}=\eta \sum_{k=1}^{L_{j}} P_{j}^{k} \hat{H}_{j}^{-1}, \quad P_{j}^{k}=\left(I \otimes J_{j}^{k}\right)^{T}\left(\hat{H}_{j}^{k}\right)^{-1}\left(I \otimes J_{j}^{k}\right) \hat{H}_{j},
$$

where, now,

$$
\hat{H}_{j}^{k}=G_{0} \otimes\left(K_{0, j}^{k}+D_{0, j}^{k}\right), \quad k=1, \ldots, L_{j} .
$$

Then, $\hat{H}_{j}^{k}$ is a local version of the positive definite matrix

$$
\hat{H}_{j}=G_{0} \otimes\left(K_{0, j}+D_{0, j}\right),
$$

which is a stochastic $H(\operatorname{div})$ matrix with a deterministic weight that provides a representation of the norm

$$
\left\|c_{0}^{-\frac{1}{2}} \vec{v}\right\|_{L^{2}(D)^{2} \otimes L^{2}(\Omega)}^{2}+\|\nabla \cdot \vec{v}\|_{L^{2}(D) \otimes L^{2}(\Omega)}^{2}
$$

on $R_{j} \otimes T$. Crucially, since $G_{0}$ is diagonal, then

$$
\left(\hat{H}_{j}^{k}\right)^{-1}=G_{0}^{-1} \otimes\left(K_{0, j}^{k}+D_{0, j}^{k}\right)^{-1}
$$

is block-diagonal and computing $\hat{S}_{j} \boldsymbol{v}$ now requires $L_{j} M$ decoupled solves with matrices of dimension approximately six. Each of these solves is a trivial cost and, moreover, there are two levels of parallelism to exploit.

The analysis in $\S 7$ is valid only for the multigrid method that uses the smoother (28). Consequently, we have no theoretical bounds for the constants $\theta$ and $\bar{\theta}$ in (24) when $V$ corresponds to the modified method. However, a preliminary analysis suggests that if $c_{0}(\vec{x})$ dominates in the expression (3), the resulting multigrid approximation is efficient.

8.1. Model problem. We now solve (2) with $f=1$ using uniformly refined meshes of triangular finite elements on $D=(-1,1) \times(-1,1)$. The spatial grid consists of $d \times d$ squares each of which is further subdivided into two equal triangles. The random diffusion coefficient $c(\vec{x}, \vec{\xi}(\omega))^{-1}$ is chosen to be of the form (3) with the covariance function

$$
C(\vec{x}, \vec{y})=\sigma^{2} \exp \left(-\left|x_{1}-y_{1}\right|-\left|x_{2}-y_{2}\right|\right) .
$$

Note that the eigenvalues and eigenfunctions of this function are known explicitly (see [12]). The random variables $\left\{\xi_{r}\right\}_{r=1}^{m}$ in (3) are assumed to be independent and 
identically distributed with zero mean and unit variance. If uniform random variables are selected, then $\Omega=[-\sqrt{3}, \sqrt{3}]^{m}$ and multivariate Legendre polynomials of total degree $n$ are used to construct the polynomial chaos basis for $T$. Alternatively, if Gaussian random variables are selected, then $\Omega=\mathbb{R}^{m}$ and multivariate Hermite polynomials are employed.

We investigate the performance of the preconditioner (25) with respect to the discretization parameters $h$ and $n$, the number of random variables $m$, and the mean $c_{0}$ and variance $\sigma^{2}$ of $c^{-1}$. In each experiment, we report preconditioned MINRES iteration counts and assess the efficiency of the multigrid approximation by computing the constants $\theta$ and $\bar{\theta}$ from (24). In each MINRES iteration, a single $\mathrm{V}$-cycle of multigrid is applied with a single pre- and post-smoothing step per grid level. The iteration is terminated when the relative residual reaches a tolerance of $10^{-6}$. All experiments were performed on a modest single-processor Linux machine with 2GB RAM.

8.2. Robustness with respect to discretization parameters. First we fix the mean and standard deviation of $c(\vec{x}, \vec{\xi}(\omega))^{-1}$ to be $c_{0}=1$ and $\sigma=0.1$. Iteration counts for solving this problem, using two types of random variables, are listed in Tables 1 and 2. The numbers in parentheses in Table 1 indicate iterations obtained with the exact preconditioner (23).

TAble 1. Preconditioned minRes iterations using multigrid with original smoother

\begin{tabular}{|l|c|cccc|cccc|}
\hline & & \multicolumn{5}{|c|}{ Uniform } & \multicolumn{4}{|c|}{ Gaussian } \\
& $n$ & $m=1$ & $m=2$ & $m=3$ & $m=4$ & $m=1$ & $m=2$ & $m=3$ & $m=4$ \\
\hline$d=16$ & 1 & $17(5)$ & $17(5)$ & $17(5)$ & $17(5)$ & $17(5)$ & $17(5)$ & $17(5)$ & $17(5)$ \\
- & 2 & $17(5)$ & $17(5)$ & $17(5)$ & $17(5)$ & $17(5)$ & $17(5)$ & $17(5)$ & $17(5)$ \\
- & 3 & $17(5)$ & $17(5)$ & $17(5)$ & $17(5)$ & $17(5)$ & $17(5)$ & $17(5)$ & $17(6)$ \\
- & 4 & $17(5)$ & $17(5)$ & $17(5)$ & $17(5)$ & $17(5)$ & $17(5)$ & $17(5)$ & $17(5)$ \\
\hline$d=32$ & 1 & $17(5)$ & $17(5)$ & $17(5)$ & $17(5)$ & $17(5)$ & $17(5)$ & $17(5)$ & $17(5)$ \\
- & 2 & $17(5)$ & $17(5)$ & $17(5)$ & $17(5)$ & $17(5)$ & $17(5)$ & $17(5)$ & $17(5)$ \\
- & 3 & $17(5)$ & $17(5)$ & $17(5)$ & $17(5)$ & $17(5)$ & $17(5)$ & $17(6)$ & $17(5)$ \\
- & 4 & $17(5)$ & $17(5)$ & $17(5)$ & $17(5)$ & $17(5)$ & $17(5)$ & $17(6)$ & $17(5)$ \\
\hline
\end{tabular}

Table 2. Preconditioned minres iterations using multigrid with modified smoother

\begin{tabular}{|l|c|cccc|cccc|}
\hline & & \multicolumn{6}{|c|}{ Uniform } & \multicolumn{5}{c|}{ Gaussian } \\
& $n$ & $m=1$ & $m=2$ & $m=3$ & $m=4$ & $m=1$ & $m=2$ & $m=3$ & $m=4$ \\
\hline$d=16$ & 1 & 17 & 17 & 17 & 18 & 17 & 17 & 17 & 17 \\
- & 2 & 17 & 18 & 19 & 19 & 17 & 19 & 19 & 19 \\
- & 3 & 17 & 19 & 19 & 19 & 18 & 19 & 19 & 19 \\
- & 4 & 17 & 19 & 19 & 19 & 18 & 19 & 19 & 19 \\
\hline$d=32$ & 1 & 17 & 17 & 17 & 18 & 17 & 17 & 17 & 18 \\
- & 2 & 17 & 18 & 18 & 18 & 18 & 18 & 18 & 19 \\
- & 3 & 17 & 18 & 18 & 18 & 18 & 18 & 18 & 19 \\
- & 4 & 17 & 18 & 18 & 18 & 18 & 18 & 19 & 19 \\
\hline
\end{tabular}


In both cases the results are robust with respect to $h, n$, and $m$. In the case of Gaussian random variables, which are unbounded, condition (5) will not hold. However, for low values of the discretization parameters, the discrete problem is well-posed and our results show that in that range the method is robust. Since (5) can't be guaranteed for Gaussian random variables, we will only consider bounded uniform random variables in the following experiments. In this example, modifying the multigrid smoother also has little to no effect on the performance of the preconditioning scheme. To reinforce this, in Table 3, we list the numerically computed constants from (24) for both variants of the multigrid method.

TABLE 3. Multigrid constants, $d=8,[\theta, \bar{\theta}]$, uniform random variables

\begin{tabular}{|l|c|ccc|}
\hline & $n$ & $m=1$ & $m=2$ & $m=3$ \\
\hline Original (Uniform) & 1 & {$[0.4589,1.0000]$} & {$[0.4589,1.0000]$} & {$[0.4589,1.0000]$} \\
- & 2 & {$[0.4586,1.0000]$} & {$[0.4587,1.0000]$} & {$[0.4587,1.0000]$} \\
Original (Gaussian) & 1 & {$[0.4589,1.0000]$} & {$[0.4589,1.0000]$} & {$[0.4578,1.0000]$} \\
- & 2 & {$[0.4583,1.0000]$} & {$[0.4584,1.0000]$} & {$[0.4584,1.0000]$} \\
\hline Modified (Uniform) & 1 & {$[0.4235,1.0003]$} & {$[0.4217,1.0009]$} & {$[0.4205,1.0013]$} \\
- & 2 & {$[0.4149,1.0000]$} & {$[0.4103,1.0008]$} & {$[0.4079,1.0012]$} \\
& & & & \\
Modified (Gaussian) & 1 & {$[0.4235,1.0003]$} & {$[0.4217,1.0009]$} & {$[0.4205,1.0013]$} \\
- & 2 & {$[0.4049,1.0004]$} & {$[0.4024,1.0008]$} & {$[0.3862,1.0009]$} \\
\hline
\end{tabular}

8.3. Robustness with respect to $a_{\max }$. The experiment above is not challenging from a statistical point of view. We know from Theorems 5 and 6 that iteration counts are likely to be sensitive to the value $a_{\max }$ in (5), even if the multigrid approximation is not. Here, we vary the mean $c_{0}$ and fix $\sigma=c_{0} / 10$ so that $c_{0}$ is the dominant term in (3) and $a_{\max }=O\left(c_{0}\right)$. We fix the discretization parameters and choose uniform random variables.

TABLE 4. MINRES iterations and multigrid constants $m=4, n=2$, $d=32$

\begin{tabular}{|c|c|c|rc|rc|}
\hline$c_{0}$ & $\sigma$ & $\begin{array}{c}\text { Exact } \\
\text { iter }\end{array}$ & iter & $\begin{array}{c}\text { Original } \\
{[\theta, \bar{\theta}]}\end{array}$ & iter & \multicolumn{2}{c|}{ Modified } \\
\hline $10^{-3}$ & $10^{-4}$ & 3 & 15 & {$[0.4552,1.0000]$} & 16 & {$[0.4068,1.0008]$} \\
$10^{-2}$ & $10^{-3}$ & 3 & 16 & {$[0.4550,1.0000]$} & 16 & {$[0.4068,1.0008]$} \\
$10^{-1}$ & $10^{-2}$ & 4 & 16 & {$[0.4556,1.0000]$} & 17 & {$[0.4071,1.0008]$} \\
$10^{0}$ & $10^{-1}$ & 5 & 17 & {$[0.3956,1.0000]$} & 18 & {$[0.4103,1.0008]$} \\
$10^{1}$ & $10^{0}$ & 8 & 21 & {$[0.4864,1.0000]$} & 22 & {$[0.4387,1.0005]$} \\
$10^{2}$ & $10^{1}$ & 16 & 40 & {$[0.6453,1.0000]$} & 45 & {$[0.5990,1.0000]$} \\
$10^{3}$ & $10^{2}$ & 46 & 99 & {$[0.9172,1.0000]$} & 107 & {$[0.8660,1.0013]$} \\
\hline
\end{tabular}

The results in Table 4 tell us that both multigrid approximations improve as $c_{0} \rightarrow$ $\infty$ and neither one deteriorates as $c_{0} \rightarrow 0$. The deterioration in the MINRES iteration counts as $c_{0} \rightarrow \infty$ is not caused by a deficiency in the multigrid approximation but 
rather is a feature of the underlying exact preconditioner (23), as indicated by the eigenvalue bound established in Theorem 5. This is a known feature of the corresponding deterministic preconditioner. Ideally, (2) should be rescaled so that for the rescaled diffusion coefficient, $a_{\max } \leq 1$.

8.4. Robustness with respect to the ratio $c_{0}^{-1} \sigma$. It is known that preconditioners for stochastic finite element systems that are based on the mean-component of the diffusion coefficient are cheap but lose efficiency when $c_{0}^{-1} \sigma \rightarrow \infty$. Theorems 6 and 15 tell us that if $V$ is based on our original multigrid method, then the efficiency of (25) is affected by $a_{\max }$, and possibly $a_{\min }$, but not directly by the ratio $c_{0}^{-1} \sigma \rightarrow \infty$ (if $a_{\max }$ remains bounded). To illustrate this, we fix $c_{0}=1$ and vary only $\sigma$. We fix all the discretization parameters and choose uniform random variables.

TABle 5. MinRes iteration counts, $m=4, n=2, c_{0}=1, d=32$

\begin{tabular}{|l|ccccccc|}
\hline$\sigma / c_{0}$ & 0.1 & 0.2 & 0.3 & 0.4 & 0.5 & 0.6 & 0.7 \\
\hline Exact & 5 & 5 & 5 & 5 & 5 & 5 & 5 \\
Original & 17 & 17 & 17 & 17 & 17 & 19 & 19 \\
Modified & 18 & 20 & 21 & 24 & 28 & $*$ & $*$ \\
\hline
\end{tabular}

The iteration counts listed in Table 5 confirm that the preconditioning scheme based on the original multigrid algorithm is insensitive to $c_{0}^{-1} \sigma$. Iteration counts deteriorate, however, for the modified version and MINRES stagnates when $c_{0}^{-1} \sigma$ exceeds a certain threshold (indicated by $*$ ). This is to be expected since the matrix

$$
\sum_{r=1}^{m} \sqrt{\lambda_{r}} G_{r} \otimes K_{r}
$$

which encapsulates the deviation of the diffusion coefficient from its mean value is not taken into account in the smoothing. This causes the quality of the multigrid approximation to deteriorate. To reinforce this, we list the associated multigrid constants in Table 6 for $d=8$. These results reinforce our earlier comment that the modified method should only be considered if the deviation of $c(\vec{x}, \vec{\xi}(\omega))^{-1}$ from the mean field is small.

TABLE 6. Multigrid constants $[\theta, \bar{\theta}]$ for modified smoother, $c_{0}=1$, $d=8$

\begin{tabular}{|cl|ccc|}
\hline & $\sigma / c_{0}$ & 0.4 & 0.5 & 0.6 \\
\hline$m=2$ & $n=1$ & {$[0.2657,1.0000]$} & {$[0.2268,1.0000]$} & {$[0.1915,1.0000]$} \\
& $n=2$ & {$[0.2155,1.0000]$} & {$[0.1651,1.0000]$} & {$[0.1187,1.0000]$} \\
\hline$m=3$ & $n=1$ & {$[0.2574,1.0000]$} & {$[0.2179,1.0000]$} & {$[0.1822,1.0000]$} \\
& $n=2$ & {$[0.2072,1.0000]$} & {$[0.1529,1.0000]$} & {$[0.1035,1.0000]$} \\
\hline$m=4$ & $n=1$ & {$[0.2194,1.0000]$} & {$[0.2090,1.0000]$} & {$[0.1727,1.0000]$} \\
& $n=2$ & {$[0.1949,1.0000]$} & {$[0.1078,1.0000]$} & {$[-\mathbf{0 . 5 4 2 6}, 1.0000]$} \\
\hline
\end{tabular}




\section{ACKNOWLEDGEMENTS}

We would like to thank John Osborn, Richard Falk, Douglas Arnold, and Ragnar Winther for several useful discussions and in particular for their assistance in establishing Lemma 1.

\section{REFERENCES}

[1] Douglas N. Arnold, Richard S. Falk, and R. Winther. Preconditioning in $H$ (div) and applications. Math. Comp., 66:957-984, 1997. MR1401938 (97i:65177)

[2] Ivo Babuška, Raúl Tempone, and Georgios E. Zouraris. Galerkin finite element approximation of stochastic elliptic partial differential equations. SIAM J. Numer. Anal., 42:800-825, 2004. MR2084236 (2005h:65012)

[3] Dietrich Braess. Finite Elements: Theory, Fast Solvers, and Applications in Solid Mechanics. Cambridge University Press, second edition, 2001. MR1827293 (2001k:65002)

[4] Franco Brezzi and Michel Fortin. Mixed and Hybrid Finite Element Methods. Springer-Verlag, 1991. MR1115205 (92d:65187)

[5] Zdzisław Brzeźniak and Tomasz Zastawniak. Basic Stochastic Processes. Springer-Verlag, 1999. MR 1713252 (2000f:60002)

[6] Claudio Canuto and Tomas Kozubek. A fictitious domain approach to the numerical solution of PDES in stochastic domains. Numer. Math., 107:257-293, 2007. MR2328847|(2008h:60277)

[7] Manas K. Deb, Ivo M. Babuška, and J. Tinsley Oden. Solution of stochastic partial differential equations using Galerkin finite element techniques. Comput. Methods Appl. Mech. Engrg., 190:6359-6372, 2001. MR 1870425 (2003g:65009)

[8] Howard Elman and Darran Furnival. Solving the stochastic steady-state diffusion problem using multigrid. IMA. J. Numer. Anal., 27:675-688, 2007. MR2371827(2009b:60248)

[9] O. G. Ernst, C. E. Powell, D. J. Silvester, and E. Ullman. Efficient solvers for a linear stochastic Galerkin mixed formulation of diffusion problems with random data. SIAM Journal Sci. Comp., 31:1424-1447, 2000. MR2486837

[10] P. Frauenfelder, C. Schwab, and R. A. Todor. Finite elements for elliptic problems with stochastic coefficients. Comput. Meth. Appl. Mech. Engrg., 194:205-228, 2005. MR 2105161 (2005i:65186)

[11] Avner Friedman. Foundations of Modern Analysis. Dover, 1982. MR663003 (83h:46001)

[12] Roger G. Ghanem and Pol D. Spanos. Stochastic Finite Elements: A Spectral Approach. Springer-Verlag, 1991. MR 1083354 (91k:73102)

[13] R. Hiptmair. Multigrid method for $\mathbf{H}($ div) in three dimensions. Electron. Trans. Numer. Anal., 6:133-152, 1997. MR1615161 (99c:65232)

[14] O. P. Le Maître, O. M. Knio, B. J. Debusschere, H. N. Najm, and R. G. Ghanem. A multigrid solver for two-dimensional stochastic diffusion equations. Comput. Methods Appl. Mech. Engrg., 192:4723-4744, 2003. MR2012487 (2004h:65012)

[15] P. Vassilevski and J. Wang. Multilevel iterative methods for mixed finite element discretizations of elliptic problems. Numer. Math., 63:503-520, 1992. MR1189534 (93j:65187)

[16] C. C. Paige and M. A. Saunders. Solution of sparse indefinite systems of linear equations. SIAM J. Numer. Anal., 12:617-629, 1975. MR0383715 (52:4595)

[17] Catherine E. Powell. Parameter-free $H$ (div) preconditioning for mixed finite element formulation of diffusion problems. IMA J. Numer. Anal., 25:783-796, 2005. MR 2170523 (2007b:65113)

[18] Catherine Elizabeth Powell and David Silvester. Optimal preconditioning for Raviart-Thomas mixed formulation of second-order problems. SIAM J. Matrix Anal. Appl., 25:718-738, 2003. MR2081231 (2005h:65054)

[19] P. A. Raviart and J. M. Thomas. A mixed finite elment method for second order elliptic problems. In I. Galligani and E. Magenes, editors, Mathematical Aspects of the Finite Element Method, no. 606 in Lecture Notes in Math., pages 292-315. Springer-Verlag, New York, 1977. MR0483555 (58:3547)

[20] Wim Schoutens. Stochastic Processes and Orthogonal Polynomials. Springer-Verlag, 2000. MR.1761401 (2001f:60095)

[21] A. Toselli and 0. Widlund. Domain Decomposition Methods - Algorithms and Theory. Springer-Verlag, 2005. MR2104179 (2005g:65006) 
[22] François Treves. Topological Vector Spaces, Distributions and Kernels. Academic Press, 1967. MR0225131 (37:726)

[23] Dongbin Xiu. Generalized (Wiener-Askey) Polynomial Chaos. Ph.D. thesis, Brown University, 2004

[24] Dongbin Xiu and George Em Karniadakis. Modeling uncertainty in steady state diffusion problems via generalized polynomial chaos. Comput. Methods Appl. Engrg., 191:4927-4948, 2002. MR 1932024 (2003h:65008)

[25] C. I. Goldstein, Z. Cai and J. E. Pasciak. Multilevel iteration for mixed finite element systems with penalty. SIAM J. Sci. Comput., 14(5):1072-1088, 1993. MR.1232176 (94h:65116)

Department of Computer Science and Institute for Advanced Computer Studies, University of Maryland, College Park, Maryland 20742

E-mail address: elman@cs.umd.edu

Applied Mathematics and Scientific Computing Program, University of Maryland, College Park, Maryland 20742

Current address: National Oceanographic Centre, Southampton S014 32H

E-mail address: d.furnival@noc.soton.ac.uk

School of Mathematics, University of Manchester, Oxford Road, Manchester, M13 9PL, UNiTEd Kingdom

E-mail address: c.powell@manchester.ac.uk 